\title{
Physics Basis for a Spherical Torus Power Plant
}

\author{
S. C. Jardin ${ }^{1}$, C. E. Kessel ${ }^{1}$, J. Menard ${ }^{1}$, \\ T. K. Mau ${ }^{2}$, R. L. Miller ${ }^{2}$,

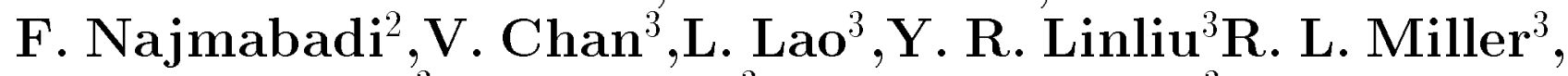 \\ T. Petrie ${ }^{3}$, P. Politzer ${ }^{3}$, A. D. Turnbull ${ }^{3}$
}

\begin{abstract}
The spherical torus, or low-aspect-ratio tokamak, is considered as the basis for a fusion power plant. A special class of wall-stabilized high- $\beta$ highbootstrap fraction low-aspect-ratio tokamak equilibrium are analyzed with respect to MHD stability, bootstrap current and external current drive, poloidal field system requirements, power and particle exhaust and plasma operating regime. Overall systems optimization leads to a choice of aspect ratio $A=1.6$, plasma elongation $\kappa=3.4$, and triangularity $\delta=0.64$. The design value for the plasma toroidal $\beta$ is $50 \%$, corresponding to $\beta_{N}=7.4$, which is $10 \%$ below the ideal stability limit. The bootstrap fraction of $99 \%$ greatly alleviates the current drive requirements, which are met by tangential neutral beam injection. The design is such that $45 \%$ of the thermal power is radiated in the plasma by Bremstrallung and trace Krypton, with Neon in the scrapeoff layer radiating the remainder.
\end{abstract}

\footnotetext{
${ }^{1}$ Princeton Plasma Physics Laboratory, P.O. Box 451, Princeton, N.J 08543

${ }^{2}$ Fusion Energy Research Program, University of California, San Diego, 9500 Gilman Dr., La Jolla, CA 92093

${ }^{3}$ General Atomics, P.O. Box 85608, San Diego, CA 92186

3
} 


\section{Introduction}

The most significant difference between the ARIES-ST optimization presently under consideration and other advanced tokamak reactor concepts studied recently, such as ARIES-RS [1], stems from the use of normal conducting toroidal field (TF) coils in ARIES-ST. For standard aspect ratio reactor designs $(\mathrm{A}>2.5)$, the dissipated power in normally conducting TF coils is prohibitively large, necessitating the use of superconducting TF coils. However, approximately 1 meter of shielding between the superconducting TF coil and the plasma on the inboard side is required to protect the superconductors from neutron damage and nuclear heating. Consequently, the designs using superconducting toroidal field coils optimize necessarily at medium to large aspect ratios, whereas designs utilizing normally conducting TF coils do not.

It was first recognized by Jassby [2] in 1977 that there were many potential engineering advantages of a copper-TF high- $\beta$ low-aspect-ratio tokamak reactor. Additional unique physics properties of this concept were described by Peng and Strickler [3], who coined the term "spherical torus", or ST. These included such desirable features as large natural elongation, strong magnetic helical pitch, and the existence of a large near-omnigeneous region in the plasma cross section which suggests improved confinement. More recent theoretical studies by Menard [4], Miller [5], and coworkers have shown that for certain specific classes of plasma equilibrium with close fitting conducting walls, there exist stable solutions to the plasma equilibrium equations with very large values of the plasma $\beta$ and with essentially all of the plasma current self-provided by the bootstrap effect. The large $\beta$ and high bootstrap fraction compensate for the low toroidal field strength and the high value of plasma current, making this concept competitive with that based on the advanced tokamak.

These theoretical results have been boosted recently by encouraging experimental results coming from the START device at Culham [7]. Although small, the START experiment has reached average $\beta$ values of $40 \%$, more than three times that what has been obtained in a tokamak of conventional aspect ratio. These values were obtained with plasma current of about $250 \mathrm{kA}, \beta_{N}=5.3, T_{\epsilon 0}=200 \mathrm{eV}, n_{e 0}=6 \times 10^{19}$, $R / a=1.31, \kappa=1.78$. with a total discharge duration time of about $20 \mathrm{~ms}$. While these high $\beta$ values have not yet been demonstrated with high bootstrap fraction, it is hoped that the longer pulse-length Mega-ampere experiments MAST [8] and NSTX [9] may be able to demonstrate this.

In the remainder of this paper we discuss the analysis that led to the physicsrelated design choices made for ARIES-ST. We present detailed analysis of MHD equilibrium, stability, and current drive requirements in high bootstrap low-aspect ratio configurations. We also present the design basis for the power and particle exhaust, plasma operating regime, and non-inductive startup requirements. 


\section{MHD Stability and Bootstrap Current}

\section{$2.1 \quad$ Overview}

Ideal MHD stability analysis of low aspect ratio tokamak plasmas has already been carried out by several authors $[4,5]$ and the relevant scalings for the maximum stable $\beta$ that might be achievable in an ST reactor are reasonably well understood. Combining Troyon $\beta$ and bootstrap current scalings, the relationship between the achievable plasma toroidal $\beta$ and the dimensionless parameters relevant to a reactor design can be expressed as:

$$
\beta(\%)=\sqrt{\epsilon} \frac{C_{B S}}{f_{B S}}\left(\frac{1+\kappa^{2}}{2}\right)\left(\frac{\beta_{N}}{2}\right)^{2} .
$$

Here $\beta$ is $2 \mu_{0}$ times the volume averaged plasma pressure divided by the vacuum toroidal magnetic field at the plasma geometric center squared, $\epsilon$ is the inverse aspect ratio, $C_{B S}$ is a bootstrap current coefficient $[6]$ typically in the range of 0.6 0.7 for the ST plasmas considered here, $\kappa$ is the plasma elongation, and $\beta_{N}$ is the normalized beta. The inefficiency of present-day non-inductive current drive sources and the large plasma currents typical of reactor-scale ST plasmas dictate that most of the plasma current must come from the neoclassical bootstrap effect. At constant aspect ratio, Eqn. 1 implies that achieving the highest possible stable $\beta$ is equivalent to simultaneously achieving the highest possible $\kappa$ and $\beta_{N}$. The strong dependence of $\beta$ on $\kappa$ and $\beta_{N}$ makes even modest improvements in either parameter important in reactor optimization. In addition, the use of a conducting wall and plasma rotation [16] and/or active feedback [17] to stabilize pressure driven kink modes is of crucial importance to the ST reactor concept, as it yields a significant increase in the stable $\beta_{N}$ limit. Such wall stabilization is assumed in nearly all of the stability analysis that follows.

\subsection{Bootstrap Current Constraint}

When the bootstrap current fraction is large, any optimization of $\beta$ must selfconsistently incorporate the bootstrap current profile in the equilibrium solution. All equilibria discussed here were generated using the JSOLVER [11] and TOO [10]fixedboundary flux-coordinate equilibrium solvers operated in such a way that all of the equilibrium current is driven by a combination of bootstrap, diamagnetic, and Pfirsch-Schlüter current with the exception of a small region near the magnetic axis. Near the magnetic axis, where the bootstrap current becomes small, two methods are used to control the current profile. The first method, described in Ref. [4], specifies a linear profile of the flux-surface-averaged parallel current density ratio

$\langle\vec{j} \cdot \vec{B}\rangle /\langle\vec{B} \cdot \nabla \phi\rangle$ between the magnetic axis and the radius of tangency to the bootstrap current profile. The value of the current density on axis is determined by the specified $q(0)$ value. The second method, described in Ref. 5, specifies that 
the surface-averaged parallel current density be the larger of the bootstrap current density and a constant value (inside some cutoff radius) determined also by a central $q(0)$ constraint. The first method ensures continuity of all equilibrium profiles but makes direct control of the bootstrap current fraction more difficult in some situations. All bootstrap current calculations use the collisionless model described in Ref. 18 and use an accurate approximate expression for the trapped particle fraction derived in Ref. 19.

\subsection{Initial Shape and Profile Optimization}

Once the equilibrium current profile is constrained to be exactly aligned with the bootstrap current, the aspect ratio, boundary shape, density and temperature profiles, and $q(0)$ completely determine the equilibrium. For analyzing the stability of these equilibria, the codes BALLOON [20, 21] and BALOO [10] were used for high-n ballooning modes, and the PEST-II [22] and GATO [23] codes were used for computation of both marginal $\beta$ values and the marginal wall position which stabilizes low-n kink modes. The functional forms used for the shape and pressure and temperature profiles in the following analysis are described in Refs. 4 and 5 and are not repeated here.

For most of the results discussed below, systematic ballooning stability optimization was performed prior to kink optimization because kink stability analysis is comparatively computationally expensive and time consuming. In this optimization, it is important to span a reasonable range of shape and profile combinations to be confident that one is truly approaching an optimal configuration. Further, the aspect ratio with the highest $\beta$ is not necessarily optimal from a power production stand-point, so determining stability limits for a range of aspect ratios is essential for reactor optimization.

The starting point for the ARIES-ST stability optimization is essentially summarized in Fig. 1 whose data is taken from Figs. 15 and 16 of Ref. [4]. The diamonds in the figure show the optimal ballooning stable $\beta_{N}$ and $\beta$ for near $100 \%$ bootstrap fraction equilibria with $q(0)=3$ and $\delta=0.45$ for a range of aspect ratios and elongation. The first important point of this figure is that increasing $\kappa$ actually leads to an increase in the marginal $\beta_{N}$ which together lead to rapidly increasing $\beta$. The second important point is that $A \geq 1.4$ is optimal for stability when the high bootstrap fraction constraint is enforced. Since the TF dissipation decreases with increasing aspect ratio, the optimal reactor configuration will also have $\mathrm{A} \geq 1.4$.

The squares in the figure represent the corresponding optimal $\beta_{N}$ and $\beta$ taken from Fig. 4 of Ref. 5 for $\kappa=2.8$ and $A=1.4$ and show a roughly $10 \%$ higher $\beta$ due either to small numerical differences between the codes or to the profile functional forms used by Miller being somewhat better suited for ballooning stability optimization. The very similar optimal pressure profiles found by the two authors are shown in Figure 2. As seen in the figure, broad pressure profiles with large gradients near the plasma edge and smaller but finite gradients in the core are optimal with respect 
to ballooning stability when the bootstrap fraction is near unity.

\subsection{Kink Stability of Ballooning Marginal Equilibria}

As mentioned above, one of the more important findings in both Refs. 4 and 5 for high bootstrap fraction equilibria optimized for ballooning stability was that the marginally stable $\beta$ increased very rapidly with increasing $\kappa$. However, it is also necessary to determine what upper limit the $n=0$ vertical instability and low- $n$ kink modes impose on $\kappa$ and hence $\beta$. The $n=1$ kink mode is found to require an increasingly close wall for stability as $\kappa$ increases and becomes unstable at zero wall separation above a certain critical $\kappa$ value. This is evident in Fig. 3 which shows the critical wall separation (normalized to the plasma minor radius) for the $n=1 \mathrm{kink}$ mode as a function of elongation for two sequences of ballooning stability optimized equilibria with aspect ratio $A=1.4$. For the equilibrium with higher triangularity $\delta=0.55$, the critical $\kappa$ is above 3.8 and decreases to approximately 3.6 as $\delta$ is reduced to 0.45 . Thus, higher elongation must be combined with higher triangularity at fixed wall separation, and higher triangularity allows a larger wall separation distance for $\mathrm{n}=1$ kink stabilization at fixed $\kappa$.

An important finding in Refs. [12], [13], and [4] also relevant to the equilibria discussed here is that intermediate- $n(3<n<10)$ kink modes often determine the minimum wall separation distance provided that $\kappa$ is not too large. This is shown in Fig. 4 which compares the conformal wall separation which marginally stabilizes $\mathrm{n}=1-6$ kink modes as a function of toroidal mode number for two ballooning stability optimized equilibria with $\mathrm{A}=1.6, \kappa=3.2$, and triangularities 0.57 and 0.35 . The $n=6$ marginal wall separation is roughly half that found for $n=1$, and a minimum separation has not been reached even at $n=6$. As discussed in Ref. 4, these intermediate-n modes appear to be destabilized by both the large pressure gradient and large (parallel) Pfirsch-Schlüter current on the outboard side of the plasma. Numerical difficulties preclude the analysis of higher-n kink modes at these low aspect ratios.

\subsection{Final Shape and Profile Optimization}

In an attempt to determine what is likely an absolute upper limit on the achievable $\beta$ in an ST reactor given the present physics understanding of ideal MHD mode stabilization, several equilibrium parameters were pushed to their physical and computational limits. First, the normalized critical wall position was chosen to be $r_{\text {wall }} / a=1.15$. Second, as discussed previously, $\kappa$ was made as large as possible while still retaining $n=1$ stability without regard to $n=0$ vertical stability. Third, in order to take advantage of the close fitting wall and in light of the trends in Fig. 4, $\delta$ was made as large as could be achieved with a free-boundary equilibrium solution of the Grad-Shafranov equation (see Section 3) using a realizable poloidal field (PF) coil set. At the highest $\delta$ values, the pressure profile specification in Ref. [5] 
is better suited to optimizing ballooning stability and is also utilized here. Lastly, another boundary shape parameter called squareness [24] was utilized in stability optimization.

As inferred from the name, the squareness parameter $\zeta$ increases the squareness (more precisely, the rectangularity when $\kappa>1$ ) of the plasma boundary. The parameter $\zeta$ can be applied separately to the inboard and outboard plasma boundary, and enters into the boundary parameterization as follows:

$$
\begin{aligned}
X(\theta) & =R_{0}+a \cos (\theta+\delta \sin \theta) \\
Z(\theta) & =\kappa a \sin (\theta+\zeta \sin 2 \theta) .
\end{aligned}
$$

Fig. 5a shows the impact of varied outboard squareness on the shape of the $A=1.6$, $\kappa=3.4, \delta=0.64$ boundary found optimal in the ARIES-ST study, and Fig. 5b shows the improvement in the ballooning marginal $\beta_{N}$ as $\zeta$ is varied from -0.1 to 0.1 . For squareness $\zeta>0.1$, the ballooning marginal $\beta_{N}$ is slowly degraded. Very similar results have been reported in Ref. 24 . In these optimizations, squareness is used primarily to improve the ballooning $\beta$ limit and has a comparatively weaker impact on kink stability.

\subsubsection{Aspect Ratio Dependence}

Early systems code studies of the reactor geometry indicated that an aspect ratio of approximately 1.6 was optimal from a cost-of-electricity (COE) standpoint. Consequently, most of the subsequent physics studies focused on plasmas with this aspect ratio. However, stability studies for aspect ratios in the range of 1.4-1.8 were also carried out using the full set of optimization techniques described in Sec. 2.5. The results for both the initial and final optimizations at three different aspect ratios are listed in Table 1. As seen in the Table, the final optimizations resulted in a $40-50 \%$ relative increase in the $\beta$ limit. Again, the increase in $\beta$ for the three aspect ratios results from a combination of effects including the use of better ballooning optimized pressure profiles, higher $\kappa$, higher $\delta$, optimal $\zeta$, and a smaller plasma-wall separation distance. All equilibria listed in Table 1 have bootstrap fractions in excess of $99 \%$.

\subsubsection{Equilibrium Parameters of the Final ARIES-ST Configuration}

As discussed in Sec. 3, a discrete poloidal field (PF) coil set with practical limits on the magnetic energy available will restrict the achievable boundary shapes for ARIES-ST. In the course of the PF coil design study, it was found that generating a free-boundary shape with positive squareness $(\zeta=0.1)$ dramatically increased the $\mathrm{PF}$ stored energy relative to shapes with zero or negative squareness. However, as seen in Fig. 5b, the impact on $\beta$ of reducing $\zeta$ below 0.1 only becomes significant when $\zeta$ is reduced below 0.0. For this reason, the equilibrium boundary of the final ARIES-ST configuration has a squareness near zero. This comes at the cost of a decrease in $\beta$ from the optimal value of $60 \%$ to $56 \%$. The resulting final ARIES-ST 
reactor configuration has $A=1.60, \kappa=3.4, \delta=0.64$. The ideal MHD stability limit for this design is $\beta=56 \%$ and $\beta_{N}=8.2$. Consistent with the other ARIES studies, we choose the operating point to be $10 \%$ below the stability limit to give some margin to reduce the likelihood of plasma disruptions. Thus, the design point is at $\beta=50 \%$ , $\beta_{N}=7.4$. and $\mathrm{f}_{\mathrm{BS}}>99 \%$. The profiles for this equilibrium are shown in Fig. 6 .

\subsection{Critical Importance of Kink-mode Suppression}

The ideal MHD stability limits found in previous sections rely heavily on the ability of a nearby conducting wall to suppress the pressure and current driven kink modes found at high $\beta_{\mathrm{N}}$ and high bootstrap fraction. Since the physics of wall stabilization is quite complex and largely unproven experimentally, it is of interest to ask what $\beta$ and bootstrap fraction can be achieved if wall stabilization of the kink mode does not turn out to be practical.

In order to obtain a kink-stable, high- $\beta$ equilibrium at low-A without a conducting wall, several modifications to the equilibrium of Fig. 6 are necessary. First, the global magnetic shear must be increased by decreasing $q(0)$ and raising $q(a)$ by increasing the triangularity. Second, $\kappa$ must be reduced to help suppress the $n=1$ kink mode. Third, the pressure gradient and Pfirsch-Schlüter current responsible for driving the modes unstable must be reduced. This is achieved by both lowering $\beta$ and by using a more peaked pressure profile which results in smaller current density near the plasma edge and a higher internal inductance. Lastly, the $99 \%$ bootstrap fraction constraint must be relaxed somewhat to accommodate the new pressure and current profiles.

The result of these modifications can be seen in Fig. 7 which shows various profiles of an $A=1.6$ equilibrium which is stable to $n=1-3 \mathrm{kink}$ and ballooning modes without wall stabilization. The equilibrium has $\kappa=3.1, \delta=0.72, \beta=29 \%, \beta_{\mathrm{N}}=5.8$, $\mathrm{f}_{\mathrm{BS}}=81 \%, l_{i}(3)=0.30$, and $p(0) /\langle p\rangle=2.0$. As is evident from comparing Fig. $7 \mathrm{~b}$ to Fig. $6 \mathrm{~b}$, the no-wall case has a significantly lower $q(0)$ and much larger magnetic shear near the plasma edge as a result of the higher triangularity and more peaked pressure and current profiles. As seen in Fig. $7 \mathrm{~d}$, the bootstrap current profile remains well-aligned at large minor radius, but $20 \%$ of the total current must now be driven in the plasma core.

The $\beta$ limit as a function of toroidal mode number for the equilibrium in Fig. 7 is shown in Fig. 8a, and it is clear that the $n=1$ kink and infinite- $n$ ballooning limits are quite similar. This indicates that this equilibrium is well-optimized with respect to ideal MHD stability. For completeness, Fig. $8 \mathrm{~b}$ illustrates that simply reducing $\beta$ for the equilibrium of Fig. 6 (keeping the geometry and profiles fixed) is insufficient to stabilize kink modes in the absence of a conducting wall. Reducing $\beta$ from $56 \%$ to $25 \%$ increases the minimum marginal $r_{\text {wall }} / a$ to 1.45 for $\mathrm{n}=3$ and the required wall separation distance apparently increases with increasing toroidal mode number above $n=3$. While this trend is significantly different than observed at higher $\beta$ as seen for example in Fig. 4, it highlights the well-known importance 
of strong global magnetic shear in kink stabilization.

\section{Poloidal Field Design and Vertical Stability}

\subsection{Poloidal Field Design}

The poloidal field (PF) coil locations and currents to provide the required plasma equilibrium are determined with free-boundary equilibrium calculations. The calculation solves the Grad-Shafranov equation subject to constraints on the poloidal magnetic flux and field at points in space. The desired outboard major radius, inboard major radius, and possibly points off the midplane are constrained to have their poloidal flux coincide with the poloidal flux on the plasma boundary. In addition, the poloidal magnetic field is constrained to be zero at the desired X-point. For the spherical torus plasmas examined in this study, the plasma parameters are extreme, with an elongation of 3.4 and triangularity of 0.65 , and an $l_{i}$ of 0.125 and $\beta$ of $56 \%$. In addition, no PF coils exist on the inboard side. These features caused considerable difficulty when using the conventional approach to free-boundary equilibrium calculations. Free-boundary equilibrium calculations are done on a rectangular grid (R,Z) because the plasma boundary, and therefore its current distribution are unknown. Rectangular grids are inherently less accurate than the flux-coordinate grids used in the MHD stability studies. The extreme plasma parameters produce profiles in the plasma that are difficult to represent on the (R,Z) grid, requiring small grid spacings. In addition, the source term on the right hand side of the Grad-Shafranov equation must be recast to obtain numerical convergence. This problem is due to two issues, the high plasma pressure and the very low $l_{i}$ value (forcing a strongly hollow toroidal current density profile).

The free-boundary equilibrium equation to be solved is given by,

$$
\begin{gathered}
\Delta^{*} \psi=-\mu_{\circ} R j_{\phi}, \\
j_{\phi}=-R \frac{d p}{d \psi}-\frac{1}{2 \mu_{o} R} \frac{d g^{2}}{d \psi} .
\end{gathered}
$$

Here $\psi$ is the poloidal flux function (equal to the poloidal magnetic flux divided by $2 \pi), j_{\phi}$ is the toroidal current density, $p$ is the plasma pressure, and $g$ is the toroidal field function (equal to $R B_{\phi}$ ). At each iteration the PF coil currents are adjusted to bring the poloidal flux at the specified boundary points equal to the poloidal flux value on the plasma boundary. At low plasma pressure the two terms in the definition of $j_{\phi}$ have the same sign and add together. However, at high pressure the toroidal field function term changes sign and the two terms cancel each other to leading order. Physically, the plasma toroidal current density is shifting to the outboard side, and becoming very small over much of the plasma cross-section. This cancellation between the two terms is critical to obtaining the proper force balance, and is not easily achieved for arbitrary choices of the functions $p(\psi)$ and $g(\psi)$. In 
addition, we are requiring a very low $l_{i}$ value, which forces $j_{\phi}$ to be strongly hollow, that is with very low value over much of the plasma cross-section and high value near the plasma edge. This causes problems for the convergence of the numerical algorithms used to solve the differential equation. The definition for $j_{\phi}$ can be recast in terms of the pressure and the parallel current density given by,

$$
j_{\phi}=-R \frac{d p}{d \psi}\left(1-\frac{B_{\phi}^{2}}{\left\langle B^{2}\right\rangle}\right)+B_{\phi} \frac{\langle j \cdot B\rangle}{\left\langle B^{2}\right\rangle} .
$$

Now both terms are always positive and therefore don't require delicate cancellations, and specifying the parallel current density avoids the toroidal current density from becoming zero on axis during the iterations. The specification of this form for $j_{\phi}$ requires surface averages to be evaluated which slows the calculation down, however, it has provided access to free boundary equilibria unobtainable by other methods. Strong relaxation of the solution during the iterations is also required for these solutions to converge.

As noted previously, the PF coil currents are determined at each iteration to satisfy our poloidal flux and field constraints, which can be described by,

$$
\left[\begin{array}{cccccc}
g_{\psi}^{11} & g_{\psi}^{12} & g_{\psi}^{13} & g_{\psi}^{14} & \ldots & g_{\psi}^{1 N_{c}} \\
g_{\psi}^{21} & g_{\psi}^{22} & g_{\psi}^{23} & g_{\psi}^{24} & \ldots & g_{\psi}^{2 N_{c}} \\
g_{B}^{31} & g_{B}^{32} & g_{B}^{33} & g_{B}^{34} & \ldots & g_{B}^{3 N_{c}} \\
g_{B}^{41} & g_{B}^{42} & g_{B}^{43} & g_{B}^{44} & \ldots & g_{B}^{4 N_{c}}
\end{array}\right]\left[\begin{array}{c}
\Delta I_{1} \\
\Delta I_{2} \\
\Delta I_{3} \\
\Delta I_{4} \\
\cdot \\
\Delta I_{N_{c}}
\end{array}\right]=\left[\begin{array}{c}
\Delta \psi_{1} \\
\Delta \psi_{2} \\
\Delta B_{3} \\
\Delta B_{4}
\end{array}\right]
$$

Here $g_{\psi}$ and $g_{B}$ represent the Green's functions relating the coil current to poloidal flux and field, respectively. The $\Delta I$ are the PF coil current corrections, and $\Delta \psi$ and $\Delta B$ are the flux and field errors. It is found that the constraint on the inboard major radius is difficult to maintain, primarily because there are no $\mathrm{PF}$ coils on the inboard side of the plasma leading to very weak influence. The smallest singular value for the matrix must be eliminated to obtain a solution. It turns out that the plasma inboard major radius can only be maintained by controlling the current distribution $\left(l_{i}\right)$ and the radial position of the $\mathrm{X}$-point $\left(R_{x}\right)$. For a given $l_{i}, R_{x}$ (and therefore the triangularity) must be greater than a certain value to avoid limiter contact on the inboard side. This is shown in Fig. 9 for an earlier design point. The graph indicates that the $\mathrm{X}$-point location must be controlled to better than $0.05 \mathrm{~m}$, and that the plasma internal inductance $\left(l_{i}\right)$ must be maintained to within $\Delta l_{i}$ of 0.015 , or about $10 \%$.

From the free-boundary equilibrium calculations it is found that there are 3 distinct poloidal flux geometries that can exist outside the plasma, and each of these would have different heat flux deposition characteristics. The pure limiter configuration (sometimes called the natural divertor) has the X-point located at $\mathrm{R}=0$, the plasma is limited, and the outboard flux in the scrape-off-layer (SOL) splits into two parts. One part, closest to the plasma boundary, passes over the top of the 
plasma and intersects the inboard wall, while the other extends vertically upward and strongly expands. The hybrid limiter configuration has the X-point located at $\mathrm{R}>0$, although the plasma is still limited (not connected to the $\mathrm{X}$-point), and the outboard flux in the SOL again splits into two parts. The diverted configuration has the $\mathrm{X}$-point at $\mathrm{R}>0$, and the plasma is connected to the $\mathrm{X}$-point, and the outboard flux in the SOL is pinched and strictly extends to the outboard. The divertor configuration was chosen as the reference in order to avoid the high beat fluxes on the inboard wall.

The PF coil locations are determined by specifying an allowed contour which gives the closest possible locations consistent with ports, the TF coils, neutral beam, and device maintenance. In these calculations we assume up/down symmetry and refer only to the number of coils in the upper half-plane. Initially, a large number (15) of PF coils are distributed along the contour, and the plasma equilibrium is calculated. The solution with many coils yields the lowest PF energy solution, and as the number of coils is reduced the PF energy will increase. At each step all PF coils are tested to determine which coil contributes the least to the PF energy. This coil is eliminated, and the process is repeated. The PF energy measure used is the product of the coil major radius and the square of its current. The elimination scheme is continued until the number of coils is equal to the number of constraints in the equilibrium calculation. In addition, it must be guaranteed that the proper equilibrium is produced (i.e. the plasma can not be limited). Shown in Fig. 10 is a plot of the PF energy measure as function of the number of PF coils used, determined from the elimination scheme. The PF energy measure increases significantly when the number of PF coils is reduced below 6 to 7 . Based on these results, the the number of PF coils was set to be 6 , and the solution is shown in Fig. 11 . The allowed PF coil contour was outside the TF coil, except for the divertor coils, which exist between the divertor and the TF coil.

From the MHD stability analysis it was found that higher order plasma shaping can improve the $\beta$-limit. The next order shaping parameter beyond triangularity, called squareness, was found to maximize $\beta$ at a value of about 0.1 . The freeboundary equilibrium calculations described above only prescribed the outboard major radius, inboard major radius, and location of the X-point. Therefore the rest of the plasma boundary was free to take on the shape that minimized the PF coil currents. The resulting plasma boundaries consistently had squareness values about -0.15 , with a corresponding stability $\beta$ limit that would be $25 \%$ lower(45\%). The free-boundary calculations were subsequently modified to include two additional flux constraints at points on the outboard plasma boundary above the midplane. It was then possible to obtain the desired squareness, however, the resulting PF coil currents were significantly larger than the original solution, with a PF energy measure that was nearly 10 times higher. This is illustrated in Fig. 12. In this case, the coils were still located on a contour that was outside the TF coil.

Solutions were then examined where the PF coils were located inside the TF coil (closer to the plasma) to reduce their currents. The PF coils could be mounted either 
on the back of the shield or the inside of the TF coil. The equilibrium solutions showed high order plasma boundary distortions when the PF coils were too close to the plasma (mounted behind the shield). For all the PF coil location combinations that were examined, when the coils were inside the TF coil, the squareness of 0.10 could not be achieved, which is attributed to the presence of higher order multipole field contributions. A compromise was found where the squareness could be made 0.0 (from the -0.15 original value), with the PF coils mounted on the inside of the TF coil, and with reasonable coil currents. The maximum stable $\beta$ value was thus reduced from $60 \%$ (for a squareness of 0.10 ) to $56 \%$. The final PF coil free-boundary equilibrium is shown in Fig. 13

\subsection{Plasma Vertical Stability and Control}

The vertical stability analysis was difficult due to the extreme plasma elongation combined with very high $\beta$ and very low $l_{i}$. The vertical instability growth rate could only be inferred from a number of analyses. The vertical position feedback control power could be estimated, but not directly simulated due to numerical difficulties for these plasmas.

The natural elongation is the elongation that a plasma takes on when it is in a strictly uniform vertical field. To elongate the plasma beyond this value a field curvature must be introduced, which then makes the plasma vertically unstable. At typical aspect ratios $(A=3.0-5.0)$ the natural elongation is very close to one. However, as the aspect ratio is decreased below 2.0, it can increase significantly. In addition to elongation, the triangularity and higher order shaping (such as squareness) also increase. How much these shape parameters increase as the aspect ratio is decreased depends strongly on the current density profile $\left(l_{i}\right)$ and pressure $\left(\beta_{p}\right)$. To a lesser extent, the details of the profiles can alter these values. At the ARIES-ST parameters, the natural elongation is in the range of 1.8-1.9. Since we require an elongation of 3.4, we must add a destabilizing field, making the plasma vertically unstable in the absence of a nearby conducting wall and active feedback system.

Several attempts at the vertical stability analysis were done. Ideal MHD stability with a perfectly conducting wall indicated that for a plasma similar to the ARIESST case, the poloidally continuous wall could be located at 0.6 times the minor radius (measured from the plasma boundary). This estimate is an upper bound because an actual resistive wall could not be located at that location, because the corresponding growth rate would be very high. If the vertical instability growth rate is plotted against the distance of a finite resistivity wall, measured from the plasma boundary and normalized to the plasma minor radius, one will observe that as the resistive wall is moved further from the plasma the vertical instability growth rate increases. At first the growth rate increases slowly, but eventually is rises very rapidly, asymptoting to infinity at some wall location. This wall location turns out to be the perfectly conducting wall location. The growth rate there is very high and the sensitivity of the growth rate to the wall location is extreme. A realistic wall 
location would be at the knee in the curve, and corresponds to a distance from the plasma that is about $70 \%$ of the perfectly conducting wall separation. Thus ideal stability analysis with a perfectly conducting wall around the plasma is considered only of qualitative use for the vertical instability.

Another analysis was used where the plasma is ideal, but the wall is treated resistively, using TEQ[14]. Unfortunately, the actual plasma shape and profile combinations could not be obtained simultaneously for this analysis. However, extensive calculations of actual structure geometries were performed with plasmas approximating the ARIES-ST case as closely as possible. Both the structure model and the growth rate results are shown in Fig. 14, for a few different combinations of $\beta_{p}$ and $l_{i}$. These results were used to infer that the plasma could be effectively slowed down by the tungsten structures to allow for a feedback control, and the growth rates were used to determined the currents and voltages necessary in the feedback coils. Based on a random vertical disturbance to the plasma of $1.0 \mathrm{~cm}$, and a plasma growth time of $70 \mathrm{~ms}$, the required peak power required for vertical position control is estimated to be 105 MVA. The feedback coils would be located just behind the shield at about 45 degrees from the outboard midplane.

A further analysis was pursued where the plasma and structure are both treated resistively, through full nonlinear dynamic simulations in the Tokamak Simulation Code (TSC)[15]. Again, there were considerable difficulties in evolving the extreme plasma current profile, and the associated safety factor profile. However, these calculations did show that the plasma vertical instability was evolving on a resistive time scale, which infers that the structures are indeed influencing the instability. In addition, the dynamic simulation showed the highly non-rigid behavior of the instability at such high elongation. Shown in Fig. 15 are a sequence of plasma boundaries as the plasma is allowed to drift vertically with no feedback control. The plasma magnetic axis drifts $2.5 \mathrm{~cm}$, while the lower plasma boundary drifts 250.0 $\mathrm{cm}$. Thus rigid displacement analysis, in which all points in the plasma are assumed to move vertically together, would under-estimate the stability requirements.

In conclusion, the vertical stability and control analysis was hampered by the inability to do the analysis at these extreme plasma parameters, particularly the large $\beta$ and very broad current profile. Analysis was performed with parameters as close as possible, and the final results were inferred from them. Thus it can not be guaranteed that the plasma would not require more extensive passive stabilization and/or more feedback control power.

\section{Current Drive}

\subsection{Introduction}

The ARIES-ST design is based on plasma equilibria that have low aspect ratio $(1.4 \leq A \leq 1.8)$, high values of plasma $\beta$ and $\epsilon \beta_{p}$, and a high pressure-driven current 
fraction $\left(f_{\nabla p}>0.95\right)$. In these equilibria, the pressure profiles are optimized to yield a self-driven current density profile that is perfectly aligned with the equilibrium current, thus minimizing the requirement for seed current drive. Typically, equilibria with $f_{\nabla p}>0.99$ have been used, where near the magnetic axis the conventional bootstrap current falls to zero and, in principle, only a small amount of seed current needs to be driven.

It is clear that optimistic assumptions have been made in constructing the high performance equilibria on which ARIES-ST is based. We first note that the achievable plasma $\beta$ and the self-driven current fraction, $f_{\nabla p}$, are sensitive to the detailed pressure gradient profile, and other global shape factors such as $\kappa$ and $\delta$. Thus, in actual reactor operation and without any external control, the density and temperature profiles will likely evolve towards an equilibrium that does not result in the desired optimal plasma performance. In our ARIES-ST design, we adopt a prudent approach by allowing for external current profile control that is capable of driving a maximum of $5 \%$ of the plasma current, in order to maintain MHD stability to ballooning and kink modes at high $\beta_{N}$. To define the associated system requirements, we further limit our control capability to driving current with a profile similar to that of the bootstrap current at the desired (or target) equilibrium. For typical ARIES-ST equilibria that have been studied, this implies a driven current profile that is peaked at $\hat{\psi}=0.8$, with $\hat{\psi}$ being the normalized poloidal flux.

We note here that plasma density profile control provides an alternative to achieving the desired pressure profile for optimum reactor operation. This can be in the form of programmed fuel pellet injection or the use of ion Bernstein wave to set up local density barriers. To design such a scheme requires a thorough knowledge of plasma transport and barrier formation, which are not yet fully developed. Also, we cannot exclude the possibility that density profile control of the desired precision may not be possible. We are thus left with the choice of current profile control by external means.

\subsection{Overview Of System Selection}

In this section, we identify non-inductive techniques that drive currents on axis and off axis near the plasma edge, and determine the reference systems for ARIESST. The criteria for selection are: modest power requirements, associated in-vessel components compatible with the fusion power core, sufficiently strong data base, and applicability during the startup phase. An important consideration is the capability of these systems to generate a plasma toroidal rotation that is sufficient to stabilize the low-n kink modes with a power level consistent with current drive.

\subsubsection{Options for On-Axis Drive}

The possibilities of driving an on-axis seed current with radio frequency (RF) techniques in a spherical torus have been investigated previously. [25, 26, 27] Since the 
amount of driven current is small $\left(<1 \%\right.$ of $I_{p}$ ), current drive efficiency is not a crucial consideration. Instead, penetration of the launched wave to the magnetic axis is the main issue here. The reason is twofold: (i) the on-axis magnetic field is relatively low $(|B| \sim 2 \mathrm{~T})$ compared to conventional tokamaks, and (ii) the strong paramagnetism of the equilibrium leads to a non-monotonic dependence of $|B|$ on $R$, the major radius along the equatorial plane, as shown in Fig. 16 for a typical ARIES-ST equilibrium. In the high end of the frequency range, the accessibility criteria for electron cyclotron (EC) waves are given by:

$$
n_{e, 13}<0.97 B_{T}^{2}
$$

for the O-mode, and

$$
n_{e, 13}<0.97 \ell(\ell+1) B_{T}^{2}
$$

for the X-mode. Here, $n_{e, 13}$ is the electron density in $10^{13} \mathrm{~cm}^{-3}, B_{T}$ is the magnetic field in Teslas, and $\ell=f / f_{c e}$ is the electron cyclotron harmonic number. As one can see, at $B_{T} \sim 2 \mathrm{~T}$, accessibility of the $\mathrm{EC}$ waves to the axis requires the central density to be well below $10^{14} \mathrm{~cm}^{-3}$, which is outside the ARIES-ST regime. For the $\mathrm{X}$-mode, one can raise the frequency (or $\ell$ ) sufficiently and launch the wave from the top of the plasma cross section towards the axis to avoid off-axis absorption. However, at higher harmonics, the wave would be very weakly damped in its initial radial transit, and scattered off the magnetic axis on subsequent reflections from the edge. Therefore EC waves can only be considered for auxiliary heating in the startup phase and off-axis current profile control at steady state.

For lower hybrid ( $\mathrm{LH}$ ) waves, the accessibility condition is

$$
N_{\|}^{2}>1+f_{p e}^{2} / f_{c e}^{2}
$$

where $N_{\|} \equiv c k_{\|} / \omega$ is the parallel wave refractive index, and $f_{p e}$ and $f_{c e}$ are the electron plasma and cyclotron frequencies, respectively. Using $B_{T}=2.6 \mathrm{~T}$ and $n_{e 0}=2.62 \times 10^{14} \mathrm{~cm}^{-3}$, we have $N_{\|}>5.0$. To avoid strong electron Landau damping at the edge, we need $N_{\|}<7.0 / T_{\epsilon 0}^{1 / 2}$ where $T_{\epsilon 0}$ is central electron temperature in units of keV. Using $T_{e 0}=20 \mathrm{keV}$, this condition translates to $N_{\|}<1.6$. Therefore, in the ARIES-ST regime, there is no window in $N_{\|}$spectrum for the LH waves to penetrate to the magnetic axis.

With regard to the fast magnetosonic wave, we look at scenarios that avoid or minimize ion cyclotron absorption in the plasma cross section, since current drive via electron absorption of the wave is the goal. In Fig. 17, the lower ion cyclotron harmonic frequencies together with the DT hybrid resonance are shown along the plasma midplane for an example $\mathrm{A}=1.6$ equilibrium where $R=3.84 \mathrm{~m}$ and $B_{0}=2.6 \mathrm{~T}$. Although not indicated in the figure, the presence of thermal and energetic alpha particles in the plasma can be taken into account by noting that $f_{c D}=f_{c \alpha}$. We also note that, because of the lack of space on the inboard side, the 
wave power needs to be launched from the outboard edge near the equatorial plane. Because of the non-monotonic dependence of the magnetic field strength with $\mathrm{R}$, there are only two windows in frequency for on-axis drive: (i) low frequency fast wave (LFFW) with $f<9 \mathrm{MHz}$, that avoids all ion resonances, and (ii) ICRF with $f \sim 26.5 \mathrm{MHz}$, that avoids ion resonances on the outboard side.

Using the CURRAY ray tracing code [28], we have demonstrated that LFFW is capable of driving currents on axis in a single radial pass of the wave power. A previous theoretical work [26] found that the fraction of power absorbed at the Alfven resonance near the outboard edge to be negligible. In a case modeling study using an $\mathrm{A}=1.6$ equilibrium, we set $f=8.5 \mathrm{MHz}$ and $N_{\|}=2.0$, and launch the fast wave from above the outboard midplane. At a peak temperature and density of $20 \mathrm{keV}$ and $2.59 \times 10^{14} \mathrm{~cm}^{-3}$, respectively, we obtained a peaked driven current profile with an efficiency of $0.029 \mathrm{~A} / \mathrm{W}$. The key issue with LFFW current drive is the large size of the antenna, which may consist of a sparse array of half-turn loops around the entire torus, and the paucity of experimental data base.

The ICRF scheme for on-axis current drive has also been examined in the same magnetic geometry, using $\mathrm{f}=26.5 \mathrm{MHz}$ as shown in Fig. 17 between the second harmonics of deuterium and tritium. The result showed an off-axis driven current profile due to strong electron damping related to the high plasma $\beta$. With the narrow frequency window, inclusion of damping by energetic $\alpha$-particles will further impede the wave penetration. Therefore this technique has not been considered for on-axis drive.

Current drive by high harmonic fast waves (HHFW) at $f \sim 20 f_{c D}$, which will be tested on the NSTX experiment at PPPL, has been considered. [29] According to our calculations, damping of this wave is so strong that penetration to the axis is possible only for low- $\beta$ operation. Thus HHFW can only be considered for midto-edge plasma profile control at full $\beta$, and for core electron heating during part of the startup phase.

We have also considered injecting high-energy neutral beams to reach the magnetic axis and drive current there. Making use of the NFREYA beam deposition code $[30,31]$, we found that for typical ARIES-ST parameters, the required beam energy is about $5 \mathrm{MeV}$ for on-axis drive with acceptable efficiency $(\sim 0.03 \mathrm{~A} / \mathrm{W})$. Shown in Fig. 18 are driven beam current profiles for beam energies of $5 \mathrm{MeV}$ and $2 \mathrm{MeV}$, using the same injection geometry. The ST equilibrium used has $R=3.8 \mathrm{~m}$, $A=1.6, \beta=40 \%, I_{p}=35 \mathrm{MA}, B_{o}=2.6 \mathrm{~T}, T_{e o}=T_{i o}=14 \mathrm{keV}, n_{e o}=3.9 \times 10^{20} \mathrm{~m}^{-3}$, and $Z_{\text {eff }}=1.9$. It is clear from the figure that a beam energy of the order of $5 \mathrm{MeV}$ is required to produce a driven current profile peaked at the center for the seed current, while the $2 \mathrm{MeV}$ beam drives an undesirable broad profile reaching all the way to the edge. A caveat to this current drive technique is that a significant fraction of beam power is wasted in driving the broad pedestal that surrounds the driven peak on axis, as shown in Fig. 18(a). Raising the beam energy further may alleviate but not eliminate this problem. The required range of beam energies does represent a large extrapolation from the negative-ion based technology used for the $0.5 \mathrm{MeV}$ 
beams presently in operation on JT-60U.

We reviewed a theory [32] proposed recently that the bootstrap current on the magnetic axis is nonzero, on detailed analysis of the particle orbits near the axis. It is found that particle orbits close to the axis are not banana in shape, but actually are shaped like a potato, and the fraction of trapped electrons with potato orbits is not zero as $\hat{\psi}$ approaches zero, meaning that the bootstrap current does not vanish there for pressure profiles that are parabolic in $r$, i.e., $p \sim p_{o}\left(1-r^{2} / a^{2}\right) \sim p_{o}(1-\hat{\psi})$. An estimate shows that the potato bootstrap current density on-axis can be significant compared to the banana bootstrap current density in the mid-plasma region. This theory thus provides the intriguing possibility of a $100 \%$ bootstrap driven tokamak.

Based on our assessment, we adopt the reference approach of relying on the selfdriven current due to potato-like particle orbits near the magnetic axis to provide the on-axis seed current on ARIES-ST. We note that even though the "potato-orbit" current is based on well-founded theory, it remains to be unambiguously reproduced in a fully kinetic particle simulation or measured in a tokamak experiment. A backup option is thus required for on-axis drive and will be LFFW current drive, which requires large antenna structures around the torus and also needs to be demonstrated experimentally.

\subsubsection{Options for Off-Axis Drive}

Many of the on-axis current drive schemes mentioned previously are also candidates for off-axis current drive in ARIES-ST. In considering these techniques, we also look for those that can simultaneously provide toroidal rotation in off-axis locations. Starting with the RF techniques, off-axis current drive by lower hybrid waves to obtain reversed shear plasma operation have been demonstrated recently in ToreSupra [33] and JT-60U [34]. Most recently, clear indication of off-axis current drive by EC waves were reported on DIII-D [35], making this wave technique a potential candidate. At the high beta level in an $\mathrm{ST}$ reactor, high harmonic fast waves are predicted to be totally damped before reaching the inner core, making them also suitable for current drive in the plasma outer region. Among the RF current drive techniques, only ICRF fast wave has indicated clearly an ability to generate plasma rotation, as observed recently on TFTR [36], JET [37], and Alcator C-Mod [38]. Various theories have been proposed and tested against the experimental results. At this point, no unified theory has yet been found that is able to account for all of the observed rotations in terms of their directions and speeds, and their radial profiles.

For driving currents near the plasma periphery $(\hat{\psi}=0.8)$, one may consider tangential neutral beam injection at moderate beam energies. In Fig. 19, we show a typical profile of current driven by neutral beams with a beam energy of $120 \mathrm{keV}$ in an $\mathrm{ST}$ reactor plasma with $\beta=54 \%$ and $\mathrm{A}=1.6$. The major radius in this case is $3.2 \mathrm{~m}$, and the beam tangency radius is $3.8 \mathrm{~m}$, resulting in a driven profile peaked around

$\hat{\psi}=0.8$ with a CD efficiency of $0.033 \mathrm{~A} / \mathrm{W}$. This technique can be attractive in that 
NBCD is generally more efficient that RF techniques, it provides a reliable source of toroidal plasma rotation for stabilizing the kink, and the required positive-ion based beam technology is readily available. The experimental data base is substantial, and the physics understanding related to current drive and rotation generation is quite well established.

In addition, one may also consider the use of coaxial helicity injection (CHI) to drive currents near the edge of the plasma. This method has been successfully demonstrated in the HIT experiment at the University of Washington [39]. The basic idea is to apply a voltage across a toroidal ring gap at the vacuum vessel for injecting helicity into the plasma. In the HIT experiment, a current of $300 \mathrm{kA}$ has been generated. The penetration of this helicity (or current) into the core appears to rely on relaxation processes within the plasma, which are not yet well understood. It is believed that ohmic dissipation of the helicity brings the plasma into an equilibrium state with the current profile peaked in the outer part of the plasma. One merit of this technique is the ohmic-like efficiency of current generation, which is orders of magnitude better than that of the non-inductive methods. A major issue is the possible deleterious effect of plasma relaxations on the global energy confinement. In addition, the need to place insulators across the toroidal gap close to the plasma implies that extra shielding will be required to protect the insulator material from neutron bombardment, thus further complicating the fusion core design.

Our overall assessment leads to the choice of NBCD at moderate energies as the reference technique for off-axis current drive and profile control in ARIES-ST. The back-up option will be HHFW current drive, in which case a separate mechanism for generating rotation will need to be considered.

\subsection{Off-Axis Current Drive With Neutral Beams}

The main purpose of off-axis current drive on ARIES-ST is to generate a current density profile peaked at $\hat{\psi}=0.8$ and similar in shape to the bootstrap current. This capability is used to provide $5 \%$ or less of the equilibrium plasma current in the off-axis region and adequate current profile control in order to maintain a stable equilibrium leading to the desired plasma performance. Taking into consideration that a toroidal plasma rotation is needed for stabilizing the external kink, we determined that neutral beam injection is most suited for off-axis drive in ARIES-ST as its unbalanced injection scheme also gives rise to toroidal plasma rotation.

Injected beam neutrals are deposited in a plasma via impact ionization and charge exchange processes caused by collisions with plasma electrons, ions and impurities. In addition to these atomic processes, multi-step ionization that results from excitation and subsequent ionization of the beam neutrals enhances the effective ionization (or beam stopping) cross-sections [40] with increasing beam energy $\left(E_{b}\right)$ or plasma electron density $\left(n_{e}\right)$. The fast ions thus produced slow down on the background electrons and ions via classical collisional processes, thus transferring heat to the plasma. The beam ions can be lost from the plasma through prompt ion 
orbit loss, and through transport via trapping in toroidal field ripples and interaction with MHD and other instabilities. In general, deep penetration and deposition of the beam is achieved with combinations of high beam energy, low electron density, and near-perpendicular injection towards the center of the plasma.

Current drive arises whenever a particle velocity distribution is distorted by an external means in a toroidally asymmetric manner. In the case of unbalanced neutral beam injection, the current arises from the parallel velocity component of the slowing-down beam ions, but is partially offset by the neoclassical electron response current flowing in the opposite direction. The net steady-state, flux-surface-averaged current density driven by the beam is given by

$$
\left\langle j_{\|}^{N B} B\right\rangle=\left\langle j_{f \|} B\right\rangle\left[1-\frac{Z_{b}}{Z_{e f f}}\left(1-G\left(Z_{e f f}, \varepsilon\right)\right)\right]
$$

where $j_{f \|}$ is the fast ion current, $Z_{b}$ and $Z_{e f f}$ are, respectively, the beam ion charge and the effective charge of the plasma ions, and $G$ is the trapped electron correction to the Ohkawa current [42].

The fast ion current is simply calculated from the beam deposition profile by making use of the uniform field solution of the Fokker Planck equation [43] for the beam distribution function. However, this approach does not take into account the possible trapping of the fast ions, even though the effect may be small for tangential injection. It also neglects energy diffusion of the beam ions which may impact the beam current density profile. Likewise single-particle orbit loss effects are also not included. When properly included, all these effects will lead to a reduction in the current drive efficiency calculated here. For a large aspect ratio tokamak $(\varepsilon=r / R \ll 1$ ), the factor $\mathrm{G}$ in Eq. 8 is given by [41]

$$
G \simeq\left(1.55+0.85 / Z_{e f f}\right) \sqrt{\varepsilon}-\left(0.20+1.55 / Z_{e f f}\right) \varepsilon
$$

In an ST plasma where $\varepsilon$ may not be small compared to unity, the G-factor in Eq. 9 is generalized to [44]

$$
G \simeq x\left[\left(0.754+2.21 Z_{e f f}+Z_{e f f}^{2}\right)+x\left(0.348+1.243 Z_{e f f}+Z_{e f f}^{2}\right] / D,\right.
$$

with

$$
\begin{aligned}
D \equiv & 1.414 Z_{e f f}+Z_{e f f}^{2}+x\left(0.754+2.657 Z_{e f f}+2 Z_{e f f}^{2}\right) \\
& +x^{2}\left(0.348+1.243 Z_{e f f}+Z_{e f f}^{2}\right) \\
x \equiv & f_{f} /\left(1-f_{t}\right)
\end{aligned}
$$

where $f_{t}$ is the fraction of trapped particles on each flux surface.

Thus, the current drive efficiency is heavily dependent on how fast the beam ions slow down by collisions with the plasma electrons and ions, and optimizes at high $E_{b}$, high $T_{e}$, and low $n_{e}$. It is also strongly influenced by the presence of 
ions and electrons trapped in banana orbits, particularly in a collisionless plasma. Tangential injection of the beam tends to enhance the current drive efficiency as it leads to a large toroidal velocity component of the beam ions when they are born. Likewise, tilting of the beamline from the equatorial plane will reduce the current drive efficiency. For hydrogenic beams, it is clear from Eq. 8 that the direction of the driven current is always the same as the beam injection direction. The beam-driven current density profile is primarily determined by the beam energy, deposition profile and the injection angle.

The operating parameters for the neutral beam system are determined with the use of the NFREYA Monte Carlo beam deposition code [30]. The criterion is to drive a current with a profile peaked at the $\hat{\psi}=0.8$ surface at the highest possible current drive efficiency, for current profile control. We impose a technological constraint that limits the beam energy to below $150 \mathrm{keV}$, consistent with positive-ion based injection systems that are now readily available. Also we note that the lower energy beams tend to be more efficient in driving rotation under the same plasma conditions.

The target plasma is represented by the reference equilibrium with self-consistent density and temperature profiles for electrons, and thermal and energetic ion species. In the absence of ionization cross section data for high-Z atoms in NFREYA, we substitute oxygen as the impurity (instead of neon or krypton) at a concentration that results in the desired $Z_{e f f}$ value. In our reference case, at a nominal value of $Z_{\text {eff }}=2.0$, an oxygen concentration of $1.8 \%$ is required with an helium ash concentration of $10 \%$. To facilitate our calculations, we have added in NFREYA an interface to a plasma equilibrium given in the common EQDSK format, and used the G-factor in Eq. 10 to properly account for the ST magnetic geometry. In the code, the toroidal velocity component of the beam ions at birth is used to calculate the driven current, leading to results that are likely optimistic since trapped particle effects have been ignored.

In neutral beam systems based on positive ion sources, the injected beams consist of deuterons in three energy components $\left(E_{b}, E_{b} / 2, E_{b} / 3\right)$. Measurements made on the TFTR US Common Long Pulse Ion Source [45] indicated a beam power ratio of (72:19:9) for the three components. Assuming the same ion sources for ARIES-ST, and using the measured neutral particle ratios in the range from 80 to $114 \mathrm{keV}$, the beam current ratios of $(81.8: 12.6: 5.6)$ are extended to $120 \mathrm{keV}$ beams in our calculations.

The direction of the beam injection is crucial in determining the beam deposition location inside the plasma, and the resultant current drive efficiency. This is characterized by (1) $\theta_{h}$, the angle that the beam axis makes with the major radius at the outboard edge in the equatorial plane, and (2) $\theta_{v}$, the angle that the beam axis makes with the horizontal plane. For exact tangential injection at $R=R_{\text {tang }}$, $\theta_{h}$ is given by $\theta_{h 0}=\arcsin \left[R_{t a n g} /\left(R_{0}+a\right)\right]$. As indicated earlier, higher beam energies give rise to deeper power deposition and current drive in the same plasma. At a fixed and weak enough beam energy, smaller $\theta_{h}$ results in deeper penetration, and vice versa. The dependence of current drive efficiency at the same radial loca- 
tion on various combinations of $E_{b}$ and $\theta_{h}$ is somewhat more involved. The general guideline we have used in our analysis is to fix $E_{b}=120 \mathrm{keV}$ and determine $\theta_{h}$ such that the peak current density is located at $\hat{\psi}=0.8$, at the prescribed density and temperature profiles. The vertical injection angle, $\theta_{v}$, is usually set to zero for a horizontal injection scheme. However, in the ARIES-ST design, the beamline is tilted downward at $\theta_{v}=-19.4^{\circ}$ in order to accommodate the toroidal ring voltage feeders to the TF coil shell at the outboard midplane.

For an ARIES-ST equilibrium with $A=1.6, R=3.2 \mathrm{~m}, B_{0}=2.2 \mathrm{~T}, I_{p}=$ $29.7 \mathrm{MA}, \beta=54 \%, T_{e o}=20 \mathrm{keV}, n_{e o}=2.27 \times 10^{20} \mathrm{~m}^{-3}$, and $Z_{\text {eff }}=2.0$, and using $E_{b}=120 \mathrm{keV}, \theta_{h}=50^{\circ}$, and $\theta_{v}=19.4^{\circ}$, we obtain a driven current density profile shown in Fig 20. In this case, the three components of the deuterium beam are included, and the CD efficiency is $I / P=0.038 \mathrm{~A} / \mathrm{W}$, corresponding to $\gamma_{B}=$ $5.4 \times 10^{20} \mathrm{~A} / \mathrm{W} / \mathrm{m}^{2}$. For this case, the beam shine-through is practically zero.

The NB current drive requirements can vary considerably with the plasma density and temperature, and their respective profiles. In our analysis, we made a scan of the CD efficiency with the electron temperature (density) for the same plasma equilibrium and with plasma $Z_{e f f}$, for use in determining the optimum operating point of the power plant. To maintain a current profile peaked at $\hat{\psi}=0.8$, the injection angle $\theta_{h}$ needs to be reduced for deeper penetration as $T_{e}$ is decreased. The calculational results for the reference ARIES-ST equilibrium are displayed in Fig. 21. We note here the increase of $\gamma_{B}$ with $T_{e o}$ is strong because there is an attendant decrease in density in order to maintain a constant plasma beta. The dependence on $Z_{e f f}$ is not straightforward, as indicated by the leveling off or reversal

of the results going from $Z_{e f f}=2.0$ to $Z_{\text {eff }}=2.2$. Three factors as illuminated in Eq. 8 are at work in determining the CD efficiency. Higher plasma $Z_{\text {eff }}$ implies shorter collision times and lower fast ion currents $\left(j_{f} \|\right)$, lower electron return current $\left(Z_{b} / Z_{\text {eff }}\right)$, and larger neoclassical correction $(G)$ to the return current.

\subsection{Plasma Rotation Induced by Neutral Beams}

Toroidal plasma rotation is a natural consequence of tangential injection of neutral beams into a tokamak plasma, due to the transfer of angular momentum from the incident beam particles to the plasma particles. In ARIES-ST this mechanism is a key component in arresting the growth of external kink modes in the presence of conducting stabilizing shells, and can be provided by the current driving neutral beams described in the previous section. Among all external current drive techniques, neutral beam injection is the most efficient in directly imparting toroidal momentum to the plasma. This phenomenon has been observed unambiguously in numerous large tokamaks, and rotation speeds of up to several hundred $\mathrm{krads} / \mathrm{s}$ have been measured. However, the measured rotation speeds are all much lower than the values predicted by neoclassical theory of radial momentum transfer via plasma viscosity. Thus, at present, it is widely accepted that plasma momentum transport or loss is anomalous at a time scale similar to that of energy confinement. 
In the tokamak magnetic geometry, there are two mechanisms for the transfer of angular momentum from the beam to the plasma. Beam ions which are born as passing particles transfer their momentum via collisional torque as they slow down by Coulomb collisions on the background plasma. For those that are born trapped, particularly ones that are born in the outboard midplane, momentum transfer is achieved through the so-called instantaneous torque. This arises from the particle radial motion during their first orbit in the plasma, resulting in a radial electric current that produces a jxB torque in the toroidal direction. Evidence of both of these transfer mechanisms has been observed on JET in a time-dependent fashion [46], where momentum transfer via slowing down of passing particles is the dominant mechanism. For ARIES-ST, because of the orientation of the the beam injection, a sizable portion of the beam particles will be born on the outboard side of the plasma and be trapped, so that momentum transfer via the instantaneous torque should play a significant role.

A detailed one-dimensional analysis of the beam-induced rotation is outside the scope of the present ARIES-ST study. Instead, we have performed an order-ofmagnitude estimate of the generated rotation mainly to underscore such a possibility. We note that moderate energy beams $(120 \mathrm{keV})$ are quite efficient in generating rotation because of their high momentum content per unit of power. Our estimate is based on the simplifying assumption that the plasma rotates as a rigid body. Then equating the momentum generation rate to the momentum loss rate, we obtain

$$
P_{b}\left(2 m_{b} / E_{b}\right)^{1 / 2} / V_{p}\left\langle n_{i}\right\rangle \simeq m_{i}\left\langle v_{\phi}\right\rangle / \tau_{E}
$$

where $P_{b}$ is the beam power, $V_{p}$ is the plasma volume, $\left\langle v_{\phi}\right\rangle$ is the bulk plasma rotation speed, and $\tau_{E}$ is the momentum (energy) confinement time. Using a typical set of ARIES-ST parameters, $V_{p}=888 \mathrm{~m}^{3}, \tau_{E}=2.1 \mathrm{~s}, P_{b}=29 \mathrm{MW},\left\langle n_{i}\right\rangle=1.49 \times$ $10^{20} \mathrm{~m}^{-3}$, and $\mathrm{D}^{+}$beam, we found that $\left\langle v_{\phi}\right\rangle \simeq 86.5 \mathrm{~km} / \mathrm{s}$ which is equal to $3.9 \%$ of the Alfven speed. In an actual situation, we expect the beam momentum input profile to peak at the beam deposition region resulting in a locally peaked rotation speed. For a more precise quantitative assessment, a full momentum transport analysis in the radial direction needs to be carried out.

\subsection{On the Need for On-Axis Seed Current Drive}

In ARIES-ST, we propose to take advantage of the self-driven current via potato particle orbits [32] to provide the seed current on axis. Based on a recent analysis [32], because of the unique particle orbit topology in the vicinity of the magnetic axis, the variation of minor radius over the width of the orbit is significant, which gives rise to a non-vanishing trapped particle fraction there. The resultant selfdriven current on axis can be substantial enough to require no on-axis current drive from external sources. As an estimate, the ratio of potato bootstrap fraction to 
banana bootstrap fraction at normalized $\sqrt{\hat{\psi}}=0.5$ is given approximately by

$$
f_{b s}^{p} / f_{b s}^{b} \sim\left(2 q_{\circ} \rho_{e} / \delta_{o} R_{o}\right)^{1 / 3} / \sqrt{a / 2 R_{o}},
$$

where $q_{o}, \delta_{0}$ are on-axis safety factor and triangularity, respectively, $\rho_{e}$ is the local electron gyroradius, and $R_{0}$ is the major radius. Using typical ARIES-ST parameters with $q_{o}=4.8, \delta_{o} \simeq 0.32, B \simeq 2 \mathrm{~T}, T_{e} \simeq 16 \mathrm{keV}, R_{0}=3.32 \mathrm{~m}$, and $A=1.6$, we found $f_{b s}^{p} / f_{b s}^{b} \sim 20 \%$, which is quite significant.

The concept of a self-driven current on axis provides the intriguing possibility of tokamak plasma equilibria where the current is driven entirely by the bootstrap effect. The existence of such equilibria has been numerically demonstrated [32] by solving the Grad-Shafranov equation using the self-driven current as the driver.

The existence and magnitude of potato-orbit bootstrap current is a subject that is presently under intense theoretical research. It will be useful if an experiment can be carried out in the near future to unambiguously verify its presence. Until this is achieved, it will be prudent to search for an alternative approach to drive an on-axis seed current in ARIES-ST. As a result of our assessment in Sec. 4.2, low-frequency fast waves have been identified as a viable backup option.

\section{Power and Particle Exhaust}

\subsection{Overview}

For the ARIES-ST tokamak to operate successfully, its divertor protective armor must be able to tolerate high heat loading. In the following discussion, we estimate the heat loads under ARIES-ST conditions and describe design techniques for how to handle these loads. In Section 5.2 we derive an expression for evaluating the peak heat flux in both inboard and outboard divertors. In Section 5.3 we estimate the peaked heat flux based on the ARIES-ST design. In Section 5.4, we propose techniques to reduce excessive divertor heating. In Section 5.5, we discuss the problem of sputtering on the wetted surfaces. In Section 5.6, prospects for successful double-null ARIES-ST operation are examined.

\subsection{Estimation of the Peaked Heat Flux}

An exponential fall off of the heat flux is assumed such that

$$
Q_{d i v}=Q_{d i v, 0} \times \exp \left[-\frac{\left(R-R_{s}\right)}{f_{e x p} \lambda_{p}}\right]
$$

The power flow to the target is then given by

$$
P_{d i v}=2 \pi \int_{R_{s}}^{\infty} \frac{Q_{d i v} R d R}{\sin \alpha}
$$


Here, $P_{d i v}$ is the power flow at the target, $Q_{\text {div }}$ is the resulting heat flux distribution, $Q_{d i v, 0}$ is the peak heat flux at the divertor strike point, $R_{s}$ is the major radius of the divertor strike point, $\lambda_{p}$ is the midplane heat flux scrape-off length, $f_{\text {exp }}$ is the flux expansion at the divertor target, and $\alpha$ is the angle between the divertor target and the separatrix. Integrating Eq. 18 and simplifying the expression, we find that

$$
Q_{d i v, 0}=\frac{P_{d i v} \sin \alpha}{2 \pi R_{0} f_{e x p} \lambda_{p}\left(1+\frac{f_{e x p} \lambda_{p}}{R_{s}}\right)}
$$

The total power to the divertor target is estimated by

$$
P_{d i v} \approx P_{\text {input }}\left(1-f_{\text {rad }}\right) f_{o b} f_{\nabla B}\left(1-f_{p f r}\right)
$$

Where $P_{\text {input }}$ is the total power input to the plasma, $f_{\text {rad }}$ is the fraction of power that is radiated, $f_{o b}$ is the fraction of transport power flowing into the outboard scrape-off layer (SOL), $f_{\nabla B}$ is the fraction of power striking the outboard divertor in the $\nabla B$ direction, and $f_{p f r}$ is the fraction of power flowing into the private flux region. Combining Eqns. 16 and 17 we arrive at the following estimate for the peak heat flux at the divertor,

$$
Q_{d i v, 0}=\frac{P_{\text {input }}\left(1-f_{\text {rad }}\right) f_{o b} f_{\nabla B}\left(1-f_{p f r}\right) \sin \alpha}{2 \pi R_{s} f_{e x p} \lambda_{p}\left(1+\frac{f_{e x p} \lambda_{p}}{R_{0}}\right)}
$$

The peak heat flux predictions from this equation have been compared with DIII-D data and typically agree to within $20 \%$ of the measured peak heat flux.

\subsection{Application to the ARIES-ST Peak Heat Flux Issue in the "Pure" DN Divertor Configuration}

Because of the uncertainties in the boundary and SOL physics of high power, doublenull divertors, care must be taken in applying Eq. 19. The mechanism (or mechanisms) responsible for determining SOL properties in double-null divertors are at present not well understood. Thus, it becomes necessary to look to data from present day double-null tokamaks for guidance. For example, for low triangularity double-null divertors in DIII-D operating at low to moderate density (i.e. half the Greenwald limit), the ratio of outboard to inboard power flow is typically $5: 1$ and we will assume this power split is appropriate for ARIES-ST. In addition, the power scrape-off length $\lambda_{p}$ must also be extrapolated. For DIII-D, $\lambda_{p}$ at the outboard midplane is typically about $1 \mathrm{~cm}$, while $\lambda_{p}$ at the inboard midplane is much less than 1 $\mathrm{cm}$ and may, in fact, depend on toroidal field $B_{T}$. Based on available DIII-D data, we will assume that $\lambda_{p} \propto 1 / B_{T}$.

The calculations below are based on the ARIES-ST parameters. In what we refer to as the "pure" double-null divertor option, the heat flows into both the upper and lower divertors and there is no significant plasma contact with any other vessel 
surfaces (e.g., the centerpost). In estimating the peak heat flux under the outer divertor legs, we make the (pessimistic) assumption that radiated power from the SOL and divertors are negligible. This gives us a "worst case" scenario. Using ARIES-ST parameters to determine the peak heat flux under the outboard divertor legs, that is, $P_{\text {input }}=529 M W, f_{\text {rad }}=0.45, f_{\text {outboard } / \text { total }}=0.85, f_{\nabla B / \text { total }}=0.5, f_{p f r}=0.10, R_{0}=$ $3.6 \mathrm{~m}, f_{\text {exp }}=20$, and $\lambda_{p, o u t}=0.01 \mathrm{~m}$, we find that $Q_{d i v, 0} \approx 31.3 \mathrm{MW} / \mathrm{m}^{2}$.

We can also give an upper bound to the peak heat flux to the tiles under the inboard divertor legs. The parameters we insert into Eq. 19 are the same as above, except $R_{0}=1.8 \mathrm{~m}, f_{\text {inboard } / \text { total }}=0.15$, and $\lambda_{p, \text { in }} \approx 0.25 \mathrm{~cm}$. The peak heat flux under the inboard leg is found to be $Q_{d i v, 0} \approx 31.3 \mathrm{MW} / \mathrm{m}^{2}$.

Both inboard and outboard heat flux are greater than what might be considered acceptable, that is, $\sim 10 \mathrm{MW} / \mathrm{m}^{2}$. In the following section we propose solutions to how the peak heat flux values can be lowered to more manageable levels.

\subsection{Two Methods for Reducing Peaked Heat Flux Values}

While the simple estimates for the peak heat fluxes to both the inboard and outboard divertors are fairly high, we think that these values can be reduced to more manageable levels by siphoning some of the SOL power to the centerpost and/or by shaping the divertors for more optimal handling of the power flow.

\subsubsection{Sharing Power with the Centerpost}

Experiments on DIII-D have demonstrated that energy confinement in (ELMing) H-mode plasmas is unchanged when the plasma is lightly limited on the centerpost and that this power is distributed (nearly) uniformly along the centerpost protective armor. In principle, if the double-null separatrix is lightly limited on the centerpost armor, the entire inboard power flow can be directed to the centerpost (and away from either inboard divertor). In the "Strawman" considered in this report, the total power flow along the inboard SOL would be $\sim 44$ MW [i.e., $P_{\text {input }} \times\left(1-f_{\text {rad }}\right) \times$ $\left.f_{\text {inboard/total }}\right]$. Taking the wetted area on the centerpost as $\sim 75 \mathrm{~m}^{2}$, we estimate that $Q_{\text {div,centerpost }} \approx 0.59 \mathrm{MW} / \mathrm{m}^{2}$. Because the value of average heat flux that the centerpost can handle has been set at $1.0 \mathrm{MW} / \mathrm{m}^{2}$ and because approximately $0.56 \mathrm{MW} / \mathrm{m}^{2}$ is already resulting from the radiated power from the core plasma, then $\sim 75 \%$ of the inboard SOL power (i.e., $\sim 33 \mathrm{MW}$ ) can be directed to the centerpost armor. Thus, the power flow to the inboard divertors $(\sim 11 \mathrm{MW})$ is much reduced and the separatrix-centerpost separation at the midplane can be small.

If the centerpost could accommodate a higher heat flux than $1 \mathrm{MW} / \mathrm{m}^{2}$ (or if $P_{\text {input }}$ were lower), it might be possible to (lightly) limit the separatrix on the centerpost armor. This might not only eliminate the power flow to the inboard divertors but also reduce the peak heat flux to the outboard divertors. Again, assuming the "Strawman" parameters and assuming an exponential scrape-off dependence of the heat flux, we find that peak heat flux in the outboard divertors can be lowered to 
$\sim 10 \mathrm{MW} / \mathrm{m}^{2}$ with the average heat flux on the centerpost $\sim 2 \mathrm{MW} / \mathrm{m}^{2}$. To do this, the flux surfaces outside the separatrix defined by $R-R_{\text {edge }}<0.005 m$ on the outboard midplane intersect the wetted centerpost, where $R_{e d g e}$ is the radial coordinate of the separatrix at the outboard midplane. The flux surfaces on the midplane for $R-R_{\text {edge }}<0.005 m$ "peel off" into the outboard divertors.

\subsubsection{Dissipating Power by Divertor Shaping}

We can also reduce heat flux by making modest modifications on the divertor configuration such that the wetted area in the divertors is increased. The most practical way to do this is to reduce the angle $\alpha$ between the divertor incline and the separatrix. To estimate the value of $\alpha$, such that $Q_{\max , 0}$ does not exceed $10 \mathrm{MW} / \mathrm{m}^{2}$, we solve for $\alpha$ using Eq. 19. For the inboard divertors, this gives $\alpha \leq 17$. For the outboard divertors, we find $\alpha \leq 26$.

\subsection{Sputtering Concerns}

While the heat flux appears manageable, the role of physical and chemical sputtering is still an issue. Under high temperature plasma conditions, sputtering off the divertor plates can result in a contaminated core plasma and a significantly shortened lifetime for the plasma-exposed vessel components. The optimal material for the divertor plates (and centerpost plates) is still under consideration. However, regardless of the material selected, it is important that the temperatures of the ionand neutral particles striking that material surface be kept "low." For example, tungsten, one of the candidates considered as a viable divertor material, appears to have favorable sputtering properties if the ion temperature at the strike points can be maintained at $50 \mathrm{eV}$ [47],[48].

It is difficult to maintain an acceptably low ion temperature at the divertor strike points, if the ion temperature upstream (e.g., on the midplane separatrix of the core plasma) is "too high." We can estimate what these downstream and upstream temperatures might be along the outboard separatrix of ARIES-ST, bearing in mind the uncertainties involved in this type of calculation. We follow the 1-D transport analysis developed by Barr and Logan [49]. We assume $T_{e} \approx T_{i}$ and that the midplane edge electron density is approximately $0.45 \times 10^{20} \mathrm{~m}^{-} 3$. We find that $T_{i, d i v} \approx 60 \mathrm{eV}$ for the divertor and $T_{i, \text { mid }} \approx 95 \mathrm{eV}$ at the midplane separatrix. While these are only estimates, they do suggest that the divertor ion temperature may not be too far off from an acceptable divertor operating temperature (i.e., at least, for tungsten components) if the midplane ion temperature can be held below $100 \mathrm{eV}$.

\subsection{Conclusions}

The peak heat flux on the inboard and outboard divertors for the 529 MW input power case has been estimated to be $\sim 31$ and $\sim 16 \mathrm{MW} / \mathrm{m}^{2}$, respectively. Both 
these values are well above the handling capabilities of present day cooling technology. These heat flux estimates may serve as an upper bound for ARIES-ST, because the radiated power outside the separatrix has not been considered here. Enhancing the radiating behavior of the SOL and divertor regions would ameliorate power flow at the divertor plates, although impurity ion transport in the SOL and divertors of the double-null configuration is not well understood at present.

Two specific methods of reducing the peak flux have been outlined. The most straightforward of the two is tilting the wetted surfaces with respect to the divertor separatrix flux surface. The angles calculated to result in a peak heat flux of $10 \mathrm{MW} / \mathrm{m}^{2}$ (i.e., $\sim 17$ and $\sim 26$ for the inboard and outboard divertors, respectively) should not significantly complicate the divertor design.

The second method assumes that the double-null ARIES-ST core plasma can run close to (or on) the centerpost without an observable adverse effect on energy confinement. Moving the core plasma closer to the centerpost can reduce (perhaps, eliminate) power flow along the inboard SOL and, if the separatrix flux surface is limited on the centerpost, power flow to the outboard divertors also can be reduced. This method has been successfully tried in DIII-D for VH-mode operation [50]. This presupposes that plasma shape and location can be controlled sufficiently to do this. On the other hand, if the plasma control does result in the plasma leaning lightly on the centerpost armor, heat flux reduction on the inboard legs would be decreased "naturally."

Predicting the seriousness of the sputtering problem cannot be considered an isolated problem of only materials science or divertor physics. Minimizing the impact of material sputtering on divertor (or centerpost) protective plates depends on the kind of material used, the particle flux on the wetted surfaces, and the ion (and neutral particle) temperatures at the divertor plates. The latter two are affected by the divertor/SOL plasma (and neutral particle) transport and the plasma density and temperature upstream (e.g., at the midplane separatrix). These, in turn, are affected by the transport properties of the core plasma. Our estimates of divertor and upstream (midplane) plasma density based on simple 1-D transport modeling suggest that plasma temperatures in the SOL and divertors are at least in a range that allows one to make a plausible argument for minimizing the effect of sputtering in ARIES-ST. A thorough study of the sputtering problem in an ARIES-ST type of tokamak, however, will require a much better understanding of double-null divertor and scrape-off physics and more sophisticated analysis tools.

\section{Plasma Operating Regime}

\subsection{Energy Confinement Requirements}

Plasma particle- and power-balance calculations to establish the appropriate ion constituent fractions and the corresponding values of the Lawson parameter, $n_{i} \tau_{E}$, 
and energy confinement time, $\tau_{E}$, given by

$$
\tau_{E}=\frac{n_{i} T_{i}+n_{e} T_{e}}{\left(n_{i} T_{i} / \tau_{E_{i}}\right)+\left(n_{e} T_{e} / \tau_{E_{e}}\right)},
$$

and a ratio of $\tau_{E i} / \tau_{E e}=1$ is assumed. The plasma-energy confinement time expressed in terms of the net heating power, $P_{T R} \simeq P_{P H}\left(1-f_{R A D}\right)$, is

$$
\tau_{E}=\frac{W_{p}}{P_{P H}\left(1-f_{R A D}\right)} .
$$

Bremsstrahlung $\left(P_{B R}\right)$ and impurity line $\left(P_{\text {line }}\right)$ radiation provide the dominant radiation loss channel, with cyclotron radiation $\left(P_{C Y}\right)$ being relatively small at the high betas and low magnetic-field strengths characteristic of the ARIES-ST. If the alpha, external heating, and ohmic powers are given by $P_{2}, P_{H}$, and $P_{\omega}$, the plasmacore radiation fraction is defined by

$$
f_{R A D} \equiv \frac{P_{B R}+P_{\text {line }}+P_{C Y}}{P_{\alpha}+p_{H}+p_{\Omega}} .
$$

The value of $f_{R A D}$ for a DT plasma at $T \simeq 20 \mathrm{keV}$ with $Z_{\text {eff }} \simeq 2$ and a highly reflective $\left(P_{C Y} \simeq 0\right)$ first wall is $\simeq 0.5$, but drops below 0.2 at $T \simeq 10 \mathrm{keV}$. Once $\tau_{E}$ is known, a comparison can be made to any of a number of empirical scaling relations $[51,52]$, with the caveat that the most appropriate relation for the low-A, ST regime is not yet established experimentally. It is emphasized that a scaling law is not needed to derive any of the ARIES design points; $\tau_{E}$ is determined from the specification of target net electrical power output, $P_{E}$, and plasma gain, $Q_{p}$, that is set by the power requirements of the current-drive system. The value of $\tau_{E}$ required for a design point is compared to the various empirical scaling laws by means of a confinement multiplier, $H^{j} \equiv \tau_{E} / \tau_{E}^{j}$, where the superscript $j$ denotes the particular scaling of interest.

Several global energy-confinement scaling relations are monitored, as follows, recast in SI units. The L-mode ITER89-P scaling $[53,54,55]$ is given by

$$
\tau_{E}^{89 P}=3.8033 \times 10^{-6} I_{\phi}^{0.85} n_{e}^{0.1} B_{\phi 0}^{0.2} a^{0.3} R_{T}^{1.2} \kappa_{X}^{0.5} A_{i}^{0.5}\left[P_{T R}\right]^{-0.5},
$$

where $A_{i}$ is the atomic mass (2.5 for a nominal 50:50 D-T fuel mixture).

A more recent $\mathrm{H}$-mode database suggested the ITERH-97P scaling [57], given by

$$
\tau_{E}^{97 P}=1.082 \times 10^{-15} I_{\phi}^{0.9} n_{e}^{0.4} B_{\phi 0}^{0.2} a^{0.19} R_{T}^{1.84} \kappa_{X}^{0.92} A_{i}^{0.2}\left[P_{T R}\right]^{-0.66} .
$$

A scaling [58] that represents a sawtoothing ELMy H-mode is given by

$$
\tau_{E}^{98 H}=5.409 \times 10^{-12} n_{e}^{0.41} B_{\phi 0}^{0.08} a^{0.23} R_{T}^{1.7} \kappa_{X}^{0.67} A_{i}^{0.2}\left[P_{T R}\right]^{-0.63} .
$$

The energy-confinement-time multipliers, $H^{j}$, for the ARIES-ST relative to these representative scaling relations are summarized in Table 2. 


\subsection{POPCON Analysis}

Plasma operating contour (POPCON) plots have been generated for the ARIES-ST using the several empirical scaling relations. The results assuming the ITER89-P relation are shown in Fig. 22 . Thermally stable operation near $T_{i} \simeq 16 \mathrm{keV}$ is suggested by this result; other scaling relations might shift this zone. Figure 22 also shows that a start-up transient with increasing plasma density and temperature passing through the saddle region near $T_{i} \simeq 7 \mathrm{keV}$ requires auxiliary heating power, $P_{A U X}$, of about $45 \mathrm{MW}$.

\subsection{Plasma Density Requirements}

The following forms are assumed for the density profile for all species and the temperature profile for all thermal species,

$$
\begin{aligned}
& n(x)=\left(n_{o}-n_{s}\right)(1-x)^{\alpha_{n}}+n_{s}, \\
& T(x)=T_{o}(1-x)^{\alpha_{T}} .
\end{aligned}
$$

where $x \equiv\left(r / r_{p}\right)^{2}$ and the $\alpha$ 's are fitting constants. A non-zero edge density, $n_{s}$, is included to lower the peak heat flux and plasma temperature at the divertor plates. The profile interface with the ARIES Systems Code (ASC) for ARIES-ST designs are summarized in Table 3 . The line-average electron density of the ARIES-ST plasma slightly exceeds the Greenwald limit [59], given by

$$
\bar{n}_{G}=10^{20} I(M A) / \pi a_{p}^{2},
$$

but this is not considered a problem for reasons summarized in Ref. [56]. The separatrix density, $n_{s}$, is kept below the Borass limit [60], given by

$$
n_{B}=0.5 \times 10^{20} q_{\perp}^{0.57} B_{\phi 0}^{0.31},
$$

where the heat flux, $q_{\perp}$, is in $M W / m^{2}$ units.

\section{Current Initiation}

\subsection{Inductive Startup}

Techniques to initiate and ramp up the plasma current to full values without a center solenoid are required for ARIES-ST. RF, neutral beam and bootstrap current drive techniques all have potential for current ramp after a certain level of current has been established by some other technique [61]. Plasma current can be initiated and ramped up to modest values using the induction available from the PF coils located near the centerpost above and below the vacuum vessel (herein called the divertor coils). Design issues include not only the volt second and loop voltage capability of 
the divertor coils and power supplies, but also the vertical and horizontal control of the plasma column during ramp up and the formation of a good null prior to breakdown.

Maintaining vertical position stability during plasma startup is likely to limit the divertor coil currents and also the available loop voltage and volt seconds. Previous work on DIII-D using electron cyclotron heating (ECH) preionization and heating [62] has shown very reliable low voltage breakdown and ramp up. With an ECH (fundamental heating, high field side launch) power of about $650 \mathrm{~kW}$ applied continuously throughout the initiation and ramp up, important results from this study are:

- Reliable initiation and ramp up achieved with the loop voltage as low as 1.6 $\mathrm{V}$ (electric field about $0.15 \mathrm{~V} / \mathrm{m}$ ).

- Reliable initiation and ramp up achieved with large error fields and no null present in the vessel.

- Current channel appeared to initiate near the center of the vessel. Location of the resonance was not important.

- In this experiment, elongation was kept below 1.3 for the duration of the rampup.

- All else being constant, the current ramp rate was a linear function of the loop voltage.

To first order we should be able to scale the various inductive and resistive components of the loop voltages calculated from the DIII-D data to ARIES-ST. Following Lloyd et al. [62], the plasma current ramp rate is given by

$$
\frac{d I_{p}}{d t}=\frac{V_{l}-\left\{V_{\text {res }}+0.51 I_{p}\left[\frac{d\left(L_{i}+L_{a}\right)}{d t}\right]\right\}}{L_{i}+L_{a}} .
$$

Here $V_{l}$ is the loop voltage, $L_{i}$ is the internal inductance, $L_{a}$ is the annular inductance, and $V_{\text {res }}$ is the resistive voltage from the plasma current. Assuming the current channel profile shapes to be the same in both cases, and assuming whatever ECH heating mode is chosen will produce the same plasma parameters as the fundamental heating on DIII-D, then the inductances and the resistive voltages will scale with the major radius of the center of the current channel. Assuming that on ARIES-ST we position the current channel in the outer part of the vessel, $R_{A R I E S-S T} \approx 4.95$ and $R_{D I I I-D} \approx 1.7$, the inductances derived for the DIII-D ECH assisted startup are given in Table 4, with the values scaled to the radius of ARIES-ST.

From Eq. 30, at a loop voltage of $5.0 \mathrm{~V}$, a current ramp rate of $1.0 \mathrm{MA} / \mathrm{s}$ would be achieved on ARIES-ST. To ramp to a plasma current of 0.1 MA would take 100 $\mathrm{ms}$ and $0.5 \mathrm{Vs}$. 
The ten PF coil set used in the EFIT equilibrium model 800708.00200 for ARIESST [63] are more than adequate to provide the needed inductive drive. To achieve the triangularity and elongation required to meet the steady-state plasma performance, the divertor coil pairs ( 1 and 6 , and 2 and 7 ) must be designed to carry 32 and 15 MA, respectively. PF coil positions and currents [64] and the resulting fields and flux at the proposed location of the current channel during rampup are given in Table 5. These calculations indicate that a net current swing of less than 1.5 MA distributed judiciously between these two divertor coil pairs will provide the required $0.5 \mathrm{Vs}$. Thus, some freedom is left in choosing the PF coil currents for achieving a reasonable null prior to breakdown, and for maintaining positional stability.

To provide assurance that breakdown can be achieved reliably and ensure a minimum of resistive volt second consumption, modest ECH power at the fundamental frequency at $R=4.95 \mathrm{~m}$ will be provided.

Some detailed studies are suggested for the next design phase. Most important, a time dependent MHD model of ramp up should be developed, using the TSC code to ensure adequate position control during early phases. Pre-bias currents on the divertor coils may result in vertical or horizontal instabilities at low plasma current, which will stress the control system. The outer PF coils must be used to provide both a reasonable null prior to breakdown and positional control. The large distance between the PF coils and the plasma column will enhance the control problems. Placing the plasma column near the outer wall will provide some passive stabilization.

A backup alternative to inductive startup would be to utilize ECH startup similar to what was done on CDX-U. [65] The power required to generate enough current to create closed flux surfaces is of the same order as that needed for the bootstrap rampup described below.

\subsection{Current Rampup Using Bootstrap Overdrive}

A rampup scenario for increasing the current from $0.3 \mathrm{MA}$ to the final $31 \mathrm{MA}$ has been developed. In order to avoid axisymmetric instabilities, the discharge starts with a small, approximately circular cross-section, limited at the outboard side (Fig. 23). The minor radius is increased (and aspect ratio is reduced) until the plasma fills the width of the reactor (at about $10 \mathrm{MA}$ current). Subsequently the elongation is raised to the final value of 3.4. If the confinement is assumed to be standard H-mode throughout, a peak power of $120 \mathrm{MW}$ is needed. Restricting the external power to $50 \mathrm{MW}$ leads to a requirement for confinement control, raising the confinement multiplier by as much as $50 \%$ over H-mode during the rampup. Most of the current is provided by bootstrap current (rising from $50 \%$ at the start of the ramp to $100 \%$ at the end).

Instead of ramping the elongation along with the size, we propose a two-stage shape evolution. As before, the plasma is always in contact with the outboard limiter. It starts with fixed elongation $(\kappa=1.2)$ and triangularity $(\delta=0.2)$. The 
size is increased until the final minor radius is reached (the width of the plasma fills the limiter). Only then are the elongation and triangularity increased to the final values. The break point is at $10.8 \mathrm{MA}$. As before, in all cases parameters are adjusted to make both the plasma energy and the current constant (not necessarily a stable equilibrium). The parameters for this case are shown in Fig. 24.

Two alternative variants were developed. Either the confinement is fixed at the level required for the final state (ordinary H-mode; $H_{97}=1.051$; $\max P_{N B}=120$ MW), or the confinement multiplier is adjusted to keep the required heating power (100 kV deuterium beam) below $50 \mathrm{MW}$. The powers and factors for these variants are shown in Figs. 25 and 26. We propose using the $P_{N B} \leq 50 \mathrm{MW}$ variant as the baseline and the other as a backup.

As with the previous case, $\beta_{p}$ never approaches the equilibrium limit, and $\beta_{N}$ is a problem only at the lowest and highest current points (Fig. 27). (An arbitrary $\beta_{N}=3$ limit was set for the small plasmas; see Fig. 28).

The final plot shows the current components (bootstrap and beam-driven) for the two variants. For the smaller plasmas, a significant level of external current drive is required.

\section{Summary and Discussion}

The wall stabilized spherical torus has the potential of leading to an attractive fusionpower configuration due to the combination of high- $\beta$ and high-bootstrap-fraction. While recent ST experimental results are encouraging, there are still major research and development issues that need to be demonstrated before serious consideration can be given to this concept moving beyond the status of a physics experiment.

The ideal MHD stability calculations presented in section 2 assume highly optimized plasma profiles and complete stabilization of all external kink mode by the conducting wall. If either of these is not experimentally realizable, the critical beta for stability can drop by at least a factor of 2, severely impacting the attractiveness of the design. Also, the stability of the plasma to non-ideal modes needs to be investigated. The resistive wall mode and the neo-classical tearing modes are of particular concern.

The question of plasma control also needs to be looked into in more detail. The vertical stability and control of these very high elongation plasmas is not assured, and is very difficult to assess computationally. It is known that the arrangement of the poloidal field coils in a spherical torus implies that the control of the plasma/centerpost standoff is severely limited, and the experimental ramifications of this must be assessed. 


\section{References}

[1] S. C. Jardin, C. E. Kessel, C. G. Bathke, D. E. Ehst, T. K. Mau,F. Najmabadi, T. W. Petrie, "Physics basis for a reversed shear tokamak power plant", Fusion Engineering and Design, 38 (1997) 27

[2] D. L. Jassby, "Small-Aspect-Ratio, Small-Major-Radius Tokamak Reactors", Comments on Plasma Physics and Controlled Fusion, 3, (1977) 151

[3] Y. K. M. Peng and D. J. Strickler, "Features of Spherical Torus Plasmas", Nuclear Fusion, 26, (1986) 769.

[4] J. E. Menard, S. C. Jardin, S. M. Kaye, C. E. Kessel, J. Manickam, "Ideal MHD Stability Limits of Low Aspect Ratio Tokamak Plasmas", Nuclear Fusion, 37, (1997) 595

[5] R. L. Miller, Y. R. Lin-Liu, A. D. Turnbull, V. S. Chan, L. D. Pearlstein, O. Sauter, and L. Villard, "Stable equilibria for bootstrap- current-driven low aspect ratio tokamaks", Phys. Plasmas, 4, (1997) 1062

[6] N. Pomphrey, "Bootstrap Dependence on Plasma Profile Parameters", Princeton University, Plasma Physics Laboratory Report PPPL-2854 (August 1992) 23 pp.

[7] A. Sykes, "The Spherical Tokamak Programme at Culham," IAEA-CN69/OV2/5, 17th IAEA Fusion Energy Conference Yokohama, Japan, 19-24 October 1998 .

[8] M. Cox, "The Mega Amp Spherical Tokamak", Proceedings of the 20th SOFT Conference, Marseille, 1998

[9] M. Ono, S. Kaye, M. Peng, et al, "Exploration of Spherical Torus Physics in the NSTX Device”,IAEA-CN-69/ ICP/01(R)-EX4/1(R), 17th IAEA Fusion Energy Conference Yokohama, Japan, 19-24 October 1998.

[10] R. L. Miller, and J. W. VanDam, “Nucl. Fusion 28, 2101 (1987)

[11] J. Delucia, S. C. Jardin, and A. M. M. Todd. “An iterative metric method for solving the inverse tokamak equilibrium problem". J. Comput. Phys., 37 (1980) $183-204$.

[12] C. Kessel, J. Manickam, G. Rewoldt, et al, Phys. Rev. Lett. 72, 1212 (1994)

[13] J. Manickam, M. S. Chance, S. C. Jardin, et al, "The prospects for magnetohydrodynamic stability in advanced tokamak regimes", Phys. Plasmas, 5 (1994) 1601-1605. 
[14] S. W. Haney, L. D. Pearlstein, R. H. Bulmer, et al, "Vertical stability analysis of tokamaks using a variational procedure", Plasma Physics Reports, Vol 23, No. 9, 1997, pp. 789-804 (from Fizika Plazmy, Vol 23, No. 9, pp. 854-871).

[15] S. C. Jardin, N. Pomphrey, and J. DeLucie, "Dynamic Modeling of Transport and Positional Control of Tokamaks", Journal of Computational Physics, 66 (1986) 481-507

[16] D. J. Ward and A. Bondeson. "Stabilization of ideal modes by resistive walls in tokamaks with plasma rotation and its effect on the beta limit." Phys. Plasmas, 2 (1995) 1570-1580.

[17] R. Fitzpatrick and T. H. Jensen. "Stabilization of the resistive wall mode using a fake rotating shell." Phys. Plasmas, 3 (1996) 2641-2652.

[18] S. P. Hirshman. "Finite-aspect-ratio effects on the bootstrap current in tokamaks." Phys. Fluids, 31 (1988) 3150-3152.

[19] Y. R. Lin-Liu and R. L. Miller. "Upper and lower bounds of the effective trapped particle fraction in general tokamak equilibria." Phys. Plasmas, 2 (1995) 1666-1668.

[20] J. M. Greene and M. S. Chance, "The Second Region of Stability Against Ballooning Modes", Nuc. Fusion 21 (1981) 453.

[21] R. L. Dewar, J. Manickam, R. C. Grimm, and M. S. Chance, “n-dependence of ballooning instabilities." Nucl. Fus., 21 (1981) 493-498.

[22] R. Grimm, R. L. Dewar, and J. Manickam, "Ideal MHD stability calculations in axisymmetric toroidal coordinate systems." J. Comput. Phys., 49 (1983) 94-117.

[23] L. C. Bernard, F. J. Helton, and R. W. Moore, Comput. Phys. Commun. 24 (1981) 377 .

[24] A. D. Turnbull, Y. R. Lin-Liu, R. L. Miller, T. S. Taylor, and T. N. Todd, "Improved MHD stability through optimization of higher order moments in crosssection shape of tokamaks." Phys. Plasmas, 6 (1999) 1113-1116.

[25] M. Cox, G. Counsell, M. R. O'Brien, et al., "Heating and Current Drive in Tight Aspect Ratio Tokamaks," Abst. 21st EPS Conf. Controlled Fusion and Plasma Phys., Montpellier (1994), Vol. II, p. 1130.

[26] V. S. Chan, S. C. Chiu, Y. R. Lin-Liu, et al., "Current Drive and Profile Control in Low Aspect Ratio Tokamaks," Abst. 22nd EPS Conf. on Controlled Fusion and Plasma Phys., Bournemouth (1995), Part I, p. 377. 
[27] T. K. Mau, S. C. Jardin, C. E. Kessel, et al., "Plasma Physics Basis and Operations of the ARIES-ST Tokamak Power Plant," Proc. 17th IEEE/NPSS Symp. on Fusion Engineering, San Diego, Vol. 2 (1997) 1047.

[28] T. K. Mau, S. C. Chiu, R. W. Harvey, "Modelling of Fast Wave Current Drive in Standard and Second-Stability Bootstrapped Reactor Plasmas," Abst. EPS Top. Conf. on Radiofrequency Heating and Current Drive of Fusion Devices, Brussels, Vol. 16E (1992) 181.

[29] T. K. Mau, S. C. Chiu, "RF Current Drive Modeling for Spherical Torus Plasmas," 12th Top. Conf. on Radio Frequency Power in Plasmas, Savannah, AIP Conf. Proc. 403 (1997)243.

[30] R. H. Fowler, J. A. Holmes, J. A. Rome, “NFREYA - A Monte Carlo Beam Deposition Code for Noncircular Tokamak Plasmas," Oak Ridge National Laboratory Report ORNL/TM-6845 (1979).

[31] J. Mandrekas, "Physics Models and User's Guide for the Neutral Beam Module of the SuperCode," Georgia Institute of Technology Report GTFR-102 (1992).

[32] K. C. Shaing, A. Y. Aydemir, Y. R. Lin-Liu, R. L. Miller, "Steady State Tokamak Equilibria without Current Drive," Phys. Rev. Lett. 79 (1997) 3562.

[33] Equipe Tore Supra (presented by X. Litaudon), Plasma Phys. Contol. Fusion 3 (1996) A251.

[34] S. Ide, et al., in Fusion Energy 1996, Montreal (IAEA, Vienna, 1997), Vol. 3, p. 253.

[35] T. C. Luce, "Current Profile Modification with Electron Cyclotron Current Drive," Bull. Am. Phys. Soc. 43 (1998) 1772; to appear in Phys. Plasmas.

[36] H. Hsuan, M. Bitter, C. K. Phillips, et al., "ICH-Induced Plasma Rotation on TFTR," AIP Conf. Proc. 355 (1996) 39.

[37] L.-G. Eriksson, et al., Plasma Phys. Control. Fusion 39 (1997) 27.

[38] J. E. Rice, M. Greenwald, I. H. Hutchinson, et al., "Observations of Central Toroidal Rotation in ICRF Heated Alcator C-Mod Plasmas," Nucl. Fusion 38 (1998) 75 .

[39] B. A. Nelson, T. R. Jarboe, D. J. Orvis, et al., Phys. Rev. Lett. 72 (1994) 3666.

[40] R. K. Janev, C. D. Boley, D. E. Post, "Penetration of Energetic Neutral Beams in Fusion Plasmas," Nucl. Fusion 29 (1989) 2125.

[41] D. R. Mikkelsen, C. E. Singer, "Optimization of Steady-State Beam-driven Tokamak Reactors," Nucl. Tech/Fusion 4 (1983) 237. 
[42] T. Ohkawa, "New Methods of Driving Plasma Current in Fusion Devices," Nucl. Fusion 10 (1970) 185.

[43] J. D. Gaffey, "Energetic Ion Distribution Resulting from Neutral Injection in Tokamaks," J. Plasma Physics 16 (1976) 149.

[44] Y. R. Lin-Liu, F. L. Hinton, “Trapped Electron Correction to Beam Driven Current in General Tokamak Equilibria," Phys. Plasmas 4 (1997) 4179.

[45] H. W. Kugel, G. M. Gammel, L. R. Grisham, et al., "Measurements of Neutral Beam Species, Impurities, Spatial Divergence, Energy Dispersion, Pressure, and Reionization Using the TFTR U.S. Common Long Pulse Ion Source," Rev. Sci. Instrum. 60 (1989) 37.

[46] K.-D. Zastrow, W. G. F. Core, L.-G. Eriksson, et al., "Transfer Rates of Toroidal Angular Momentum During Neutral Beam Injection," Nucl. Fusion 38 (1998) 257.

[47] W. Eckstein and J. Laszlo, J. Nucl. Mater. 183, 19 (1991).

[48] C. H. Wu and U. Mszanowski, J. Nucl. Mater. 218, 293 (1995).

[49] W. L. Barr and B .G. Logan, Fusion Technol.18 251 (1990).

[50] G. L. Jackson, T. S. Taylor, C. J. Lasnier, et. al., Phys. Plasmas 3, 1005 (1996).

[51] “ITER Conceptual Design Interim Report," ITER Documentation Internal Series No. 7, International Atomic Energy Agency, Vienna (1989).

[52] N. A. Uckan, "Tokamak Confinement Projections and Performance Goals," Fusion Technol. 15 (March 1989) 391.

[53] P. N. Yushmanov, T. Takizuka, K. S. Riedel, O. J. W. F. Karduan, J. G. Cordey, S. M. Kaye, and D. E. Post, "Scalings for Tokamak Energy Confinement," Nuclear Fusion, 30, 10 (1990) 1999.

[54] D. E. Post, K. Borrass, J. D. Callen, S. A. Cohen, J. G. Cordey, F. Engelmann, et al., "ITER Physics," International Thermonuclear Experimental Reactor documentation series No. 21 (November 1990).

[55] S. M. Kaye, et al., "ITER L Mode Confinement Database," Nuclear Fusion, $37,9(1997) 1303$.

[56] "Technical Basis for the ITER Interim Design Report, Cost Review and Safety Analysis," ITER EDA Documentation Series No. 7, International Atomic Energy Agency, Vienna (1996). 
[57] “Technical Basis for the ITER Final Design Report, Cost Review and Safety Analysis," ITER EDA Documentation Series No. 16, International Atomic Energy Agency, Vienna (1998).

[58] N. A. Uckan, D. E. Post, and J. C. Wesley, "Physics Design Guidelines and Methodologies Derived from ITER Physics Basis," Fusion Technol., 34, 3, Part 2 (November 1998) 371.

[59] M. Greenwald, J. L. Terry, S. M. Wolfe, S. Ejima, M. G. Bell, S. M. Kaye, and G. H. Neilson, "A New Look at Density Limits in Tokamaks," Nuclear Fusion, $28,12(1988) 2199$.

[60] K. Borass, "Disruptive Tokamak Density Limit As Scrape-off Layer/Divertor Phenomenon," Nuclear Fusion, 31, 6 (1991) 1035.

[61] P.A. Politzer, ARIES-ST meeting, July 1998.

[62] B. Lloyd et al., Nuclear Fusion 31, 2031 (1991).

[63] L.L. Lao, General Atomics, private communication (1998).

[64] J.A. Leuer, General Atomics, private communication (1998).

[65] C.B. Forest, Y.S. Hwang, M. Ono, and D.S. Darrow, Phys. Rev. Lett. 68, 3559 (1992). 


\begin{tabular}{|c|c|c|c|c|c|c|}
\hline$A$ & $\kappa$ & $\delta$ & $\zeta$ & $\beta_{N}$ & $\beta_{t}(\%)$ & $r_{\text {wall }} / a$ \\
\hline \hline 1.4 & 3.4 & 0.55 & 0.000 & 7.8 & 56 & 1.21 \\
\hline 1.6 & 3.2 & 0.57 & 0.000 & 7.0 & 40 & 1.21 \\
\hline 1.8 & 3.0 & 0.57 & 0.000 & 6.6 & 31 & 1.21 \\
\hline
\end{tabular}

\begin{tabular}{c|c|c|c|c|c|c|c|}
\hline $\mathrm{A}$ & $\kappa$ & $\delta$ & $\zeta$ & $\beta_{\mathrm{N}}$ & $\beta_{\mathrm{t}}(\%)$ & $\mathrm{r}_{\text {wall }} / \mathrm{a}$ \\
\hline \hline \multirow{3}{*}{ Final: } & 1.4 & 3.6 & 0.64 & 0.065 & 8.8 & 74 & 1.16 \\
\cline { 2 - 8 } & 1.6 & 3.4 & 0.64 & 0.088 & 8.3 & 60 & 1.15 \\
\hline 1.8 & 3.2 & 0.64 & 0.088 & 7.8 & 46 & 1.16 \\
\hline
\end{tabular}

Table 1: Optimized equilibrium parameters for an ST fusion reactor. 


\begin{tabular}{lc}
\hline Scaling Relation & $H^{j}$ \\
\hline ITER89-P & 2.62 \\
ITERH-97P & 1.35 \\
ITER98-H & 1.33 \\
\hline
\end{tabular}

Table 2: ARIES-ST Energy Confinement Multipliers 
Table 3:

ARIES-ST Plasma Profiles ${ }^{(a)}$ and Other Profile Information

\begin{tabular}{lc}
\hline Parameter & Value \\
\hline Peak-to-average pressure, $p_{0} / p\left(=n_{0} T_{0} / n T\right)$ & 1.386 \\
Peak-to-average density, $n_{0} / n$ & 1.22 \\
Peak-to-average temperature, ${ }^{(b)} T_{0} / T$ & 1.136 \\
Peak-to-average temperature, ${ }^{(c)} T_{0} / T$ & 1.165 \\
Separatrix-to-average density, $n_{s} / n$ & 0.02 \\
Density profile exponent, $\alpha_{n}$ & 0.224 \\
Temperature profile exponent, $\alpha_{T}$ & 0.165 \\
Pressure profile exponent, $\alpha_{p}$ & 0.385 \\
\hline${ }^{(a)}$ Inputs to the ARIES Systems Code (ASC) & are boxed. \\
${ }^{(b)}$ The density-weighted, volume-averaged temperature. \\
(c) The volume-averaged temperature.
\end{tabular}




\begin{tabular}{|c|c|c|}
\hline & DIII-D & ARIES-ST \\
\hline$L_{i}(\mu \mathrm{H})$ & 0.5 & 1.46 \\
$L_{a}(\mu \mathrm{H})$ & 0.3 & 0.87 \\
$V_{\text {res }}(\mathrm{V})$ & 0.8 & 2.33 \\
$0.5 I_{p}\left[\frac{d\left(l_{i}+L_{a}\right)}{d t}\right](\mathrm{V})$ & 0.15 & 0.44 \\
\hline
\end{tabular}

Table 4: ARIES-ST inductances and loop voltages scaled from DIII-D assisted startup data 


\begin{tabular}{|c|c|c|c|c|c|c|}
\hline Coil No. & $R_{\text {coil }}(\mathrm{m})$ & $Z_{\text {coil }}(\mathrm{m})$ & $I_{\text {coil }}(\mathrm{MA})$ & $B_{r}(\mathrm{~T})$ & $B_{z}(\mathrm{~T})$ & Flux $(\mathrm{Wb})$ \\
\hline 1 & 2.0 & 10.5 & 31.13 & -0.028 & 0.036 & 3.76 \\
2 & 3.0 & 10.5 & -14.45 & 0.027 & -0.037 & -3.75 \\
3 & 10.5 & 7.5 & -26.52 & 0.30 & -0.77 & -62.88 \\
4 & 10.5 & 6.3 & 26.06 & -0.34 & 0.92 & 73.56 \\
5 & 10.5 & 4.3 & -16.42 & 0.24 & -0.79 & -60.77 \\
6 & 2.0 & -10.5 & 31.66 & 0.028 & 0.037 & 3.83 \\
7 & 3.0 & -10.5 & -14.48 & -0.027 & -0.037 & -3.75 \\
8 & 10.5 & -7.5 & -27.48 & -0.31 & -0.80 & -65.15 \\
9 & 10.5 & -6.3 & 26.56 & 0.34 & 0.94 & 74.98 \\
10 & 10.5 & -4.3 & -16.04 & -0.24 & -0.78 & -59.34 \\
\hline
\end{tabular}

Table 5: PF coil positions and currents for ARIES equilibrium 800708.00200 and the fields and flux at $\mathrm{R}=4.95$ and $\mathrm{Z}=0$. 
Figure 1 - Ballooning marginal stability of high bootstrap fraction equilibria for a range of aspect ratios and elongations for optimizations performed prior to the ARIES-ST reactor study. Diamonds are from Ref. 4 and squares are from Ref. 5 for $\kappa=2.8$ and $\mathrm{A}=1.4$.

Figure 2 - (a) Pressure profiles and (b) first derivatives of pressure profiles which optimize ballooni ng stability at $\mathrm{A}=1.4$ and $\kappa=2.8$ for the data shown in Fig. 1 .

Figure 3 - Conformal wall separation (normalized to the plasma minor radius) which marginally stabilizes the $n=1$ kink mode as a function of plasma elongation for a ballooning stability optimized equilibrium with $\mathrm{A}=1.4$.

Figure 4 - Conformal wall separation which marginally stabilizes $n=1-6$ kink modes as a function of toroidal mode number for ballooning stability optimized equilibria with $\mathrm{A}=1.6, \kappa=3.2$, and triangularities 0.57 and 0.35 .

Figure 5 - (a) Boundary shapes for the final ARIES-ST configuration $(A=1.6$, $\kappa=3.4, \delta=0.64$ ) with outboard squareness values $\zeta_{\text {out }}=-0.1$ (dashed), 0.0 (solid), and +0.1 (dot-dash). (b) Impact of $\zeta_{\text {out }}$ on the ballooning marginal stability $\beta$ limit for the final ARIES-ST configuration.

Figure 6 - Equilibrium details of the final ARIES-ST configuration with A=1.60, $\kappa=3.4, \delta=0.64, \beta=56 \%$ and $\mathrm{f}_{\mathrm{BS}}>99 \%$ which is marginally stable to ballooning and $\mathrm{n}=1-6$ kink modes with a conducting wall at $\mathrm{r}_{\text {wall }} / \mathrm{a}=1.15$. ARIES-ST operating point has $\beta$ and $\beta_{N}$ reduced $10 \%$ below these values. (a) poloidal flux contours, (b) safety factor profile, (c) pressure, temperature, and density profiles, (d) total current, bootstrap current, and external current drive profiles.

Figure 7 - Details of an optimized ST equilibrium with $\mathrm{A}=1.60, \kappa=3.1, \delta=0.72$, $\beta=29 \%$ and $\mathrm{f}_{\mathrm{BS}}=80 \%$ which is stable to ballooning and $\mathrm{n}=1-3 \mathrm{kink}$ modes without a conducting wall: (a) poloidal flux contours, (b) safety factor profile, (c) pressure, temperature, and density profiles, $(d)$ total current, bootstrap current, and external current drive profiles.

Figure 8 - (a) No-wall kink and ballooning marginal $\beta$ for the equilibrium of Figure 7 (b) Kink marginal wall position for an equilibrium with the same geometric parameters, profiles, and $f_{\mathrm{BS}}$ as in Figure 6 , but with $\beta_{\mathrm{N}}\left(\beta_{t}\right)$ reduced to $5.5(25 \%)$.

Figure 9 - For a given internal inductive $l_{i}$ and $\beta$, the inboard gap between the first wall and plasma boundary depends on the radial position of the x-point, $R_{x}$. Here, Here, $\mathrm{A}=1.6, \mathrm{R}=3.30 \mathrm{~m}, \mathrm{a}=2.05 \mathrm{~m}, I_{p}=32 \mathrm{MA}$.

Figure 10 - Dependence of the energy in the Poloidal Field (PF) System as a function of the number of PF coils used. Here, $A=1.6, I_{p}=32 M A, R=3.29 \mathrm{~m}$, $a=2.05 m, \kappa=3.35, \delta=0.53, \beta_{p}=1.95, l_{i}=0.175$

Figure 11 - Plasma-vacuum interface for the reference 6-coil solution. 
Figure 12 - Comparison of free boundary solutions with values of the squareness parameter $\zeta$ of -0.15 and 0.10 . Note that the PF energy increases by nearly 10 for the larger squareness value.

Figure 13 - The final ARIES-ST free boundary solution showing the plasma vacuum boundary and $\mathrm{PF}$ coil location and currents.

Figure 14 - Structure model and vertical instability growth rate results for several combinations of $\beta_{p}$ and $\ell_{i}$

Figure 15 - Sequence of plasma boundaries as the plasma is allowed to drift vertically with no feedback control. The behavior is highly non-rigid.

Figure 16 - Magnitude of magnetic field along midplane major radius in a typical $\mathrm{ST}$ reactor plasma.

Figure 17 - Selection of fast wave frequency for on-axis current drive in an ST reactor plasma.

Figure 18 - Neutral beam driven current profiles for a typical ST reactor plasma using a beam energy of (a) $2 \mathrm{MeV}$, and (b) $5 \mathrm{MeV}$.

Figure 19 - Off-axis neutral beam driven current profile for a typical ST reactor plasma using a beam energy of $120 \mathrm{keV}$.

Figure 20 - Off-axis neutral beam driven current profile in an ARIES-ST plasma using a beam energy of $120 \mathrm{keV}$ with three components.

Figure 21 - Calculated current drive efficiency as functions of electron temperature and plasma $Z_{\text {eff }}$, for ARIES-ST using $120 \mathrm{keV}$ neutral beams with current peaked at $\hat{\psi}=0.8$.

Figure 22 - ARIES-ST Plasma Operating Contour (POPCON) diagram. Solid countours indicate auxiliary heating power, $P_{A U X}$, in increments of $10 \mathrm{MW}$ until "ignition" at the dashed contours.

Figure 23 - The minor radius and elongation are increased with the plasma current. Neutral beam current drive and power requirements are shown.

Figure 24 - Geometrical quantities as a function of plasma current during bootstrap overdrive startup.

Figure 25 - Required confinement multiplier for the two startup variants.

Figure 26 - Log-Log plot of the power requirements for the two cases

Figure 27 - Stability parameters for the 2 startup variants.

Figure 28 - Log-Log plot of the current-drive requirements for the two cases. 


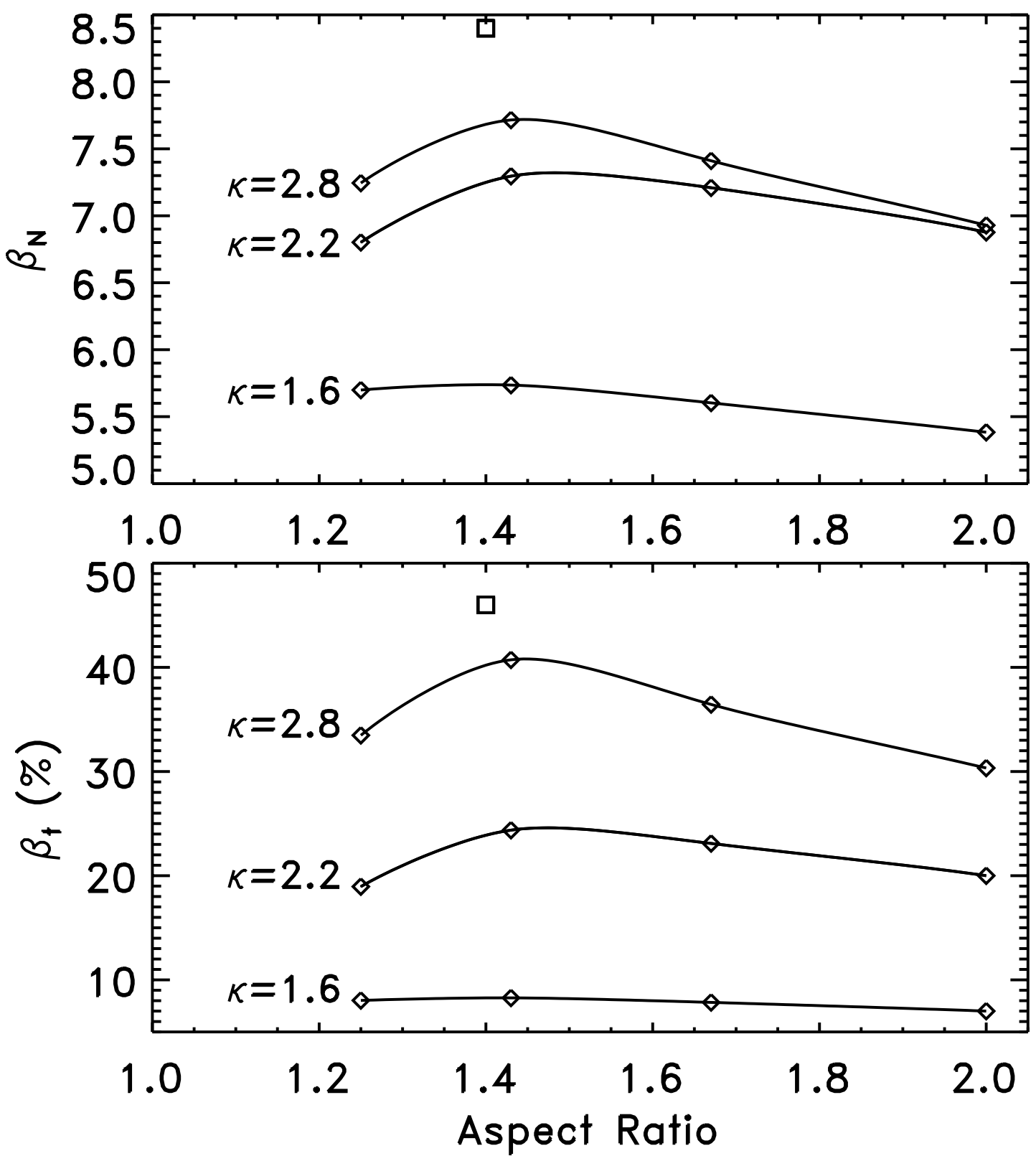

Figure 1: Ballooning marginal stability of high bootstrap fraction equilibria for a range of aspect ratios and elongations for optimizations performed prior to the ARIES-ST reactor study. Diamonds are from Ref. 4 and squares are from Ref. 5 for $\kappa=2.8$ and $\mathrm{A}=1.4$. 

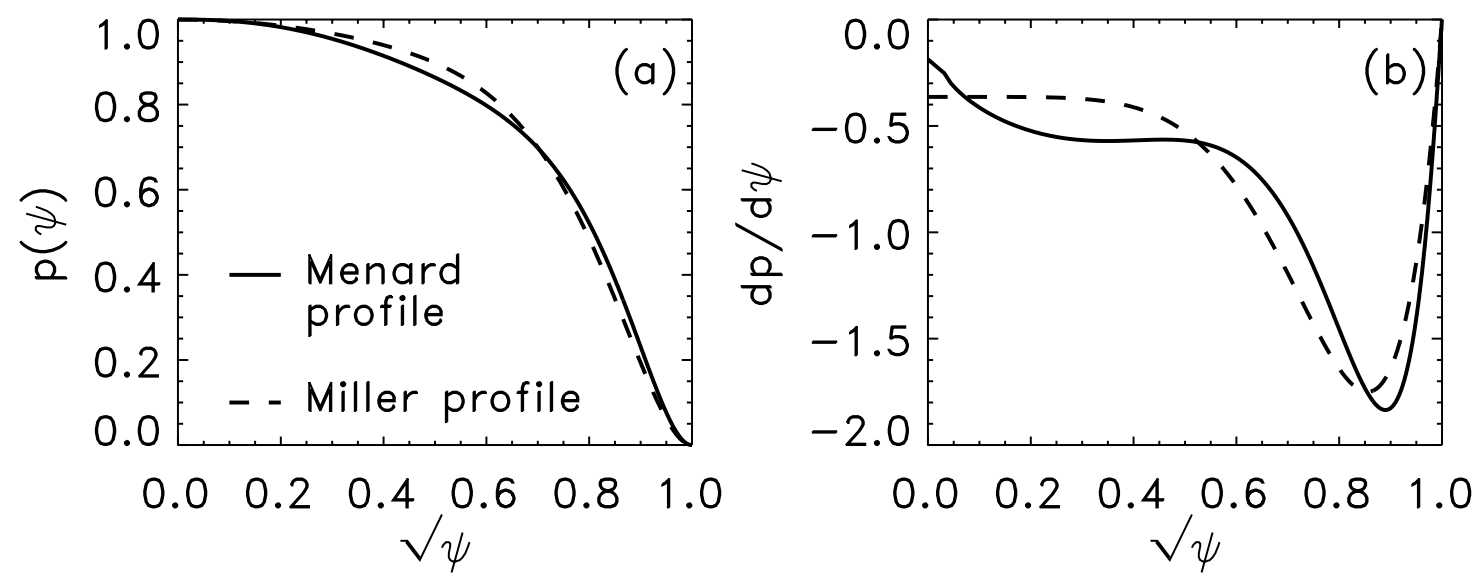

Figure 2: (a) Pressure profiles and (b) first derivatives of pressure profiles which optimize ballooning stability at $\mathrm{A}=1.4$ and $\kappa=2.8$ for the data shown in Fig. 1 . 


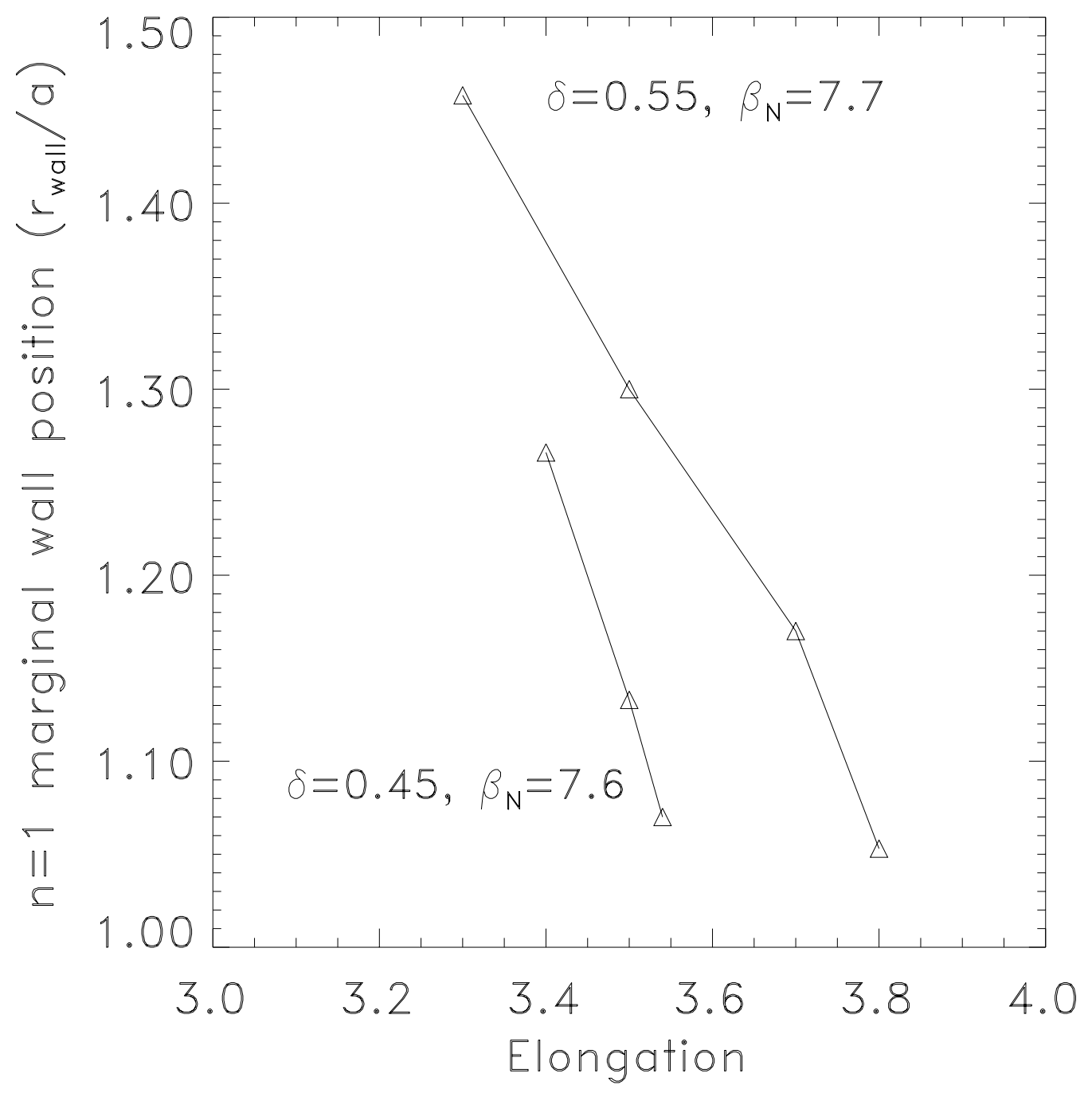

Figure 3: Conformal wall separation (normalized to the plasma minor radius) which marginally stabilizes the $n=1$ kink mode as a function of plasma elongation for a ballooning stability optimized equilibrium with $\mathrm{A}=1.4$ 


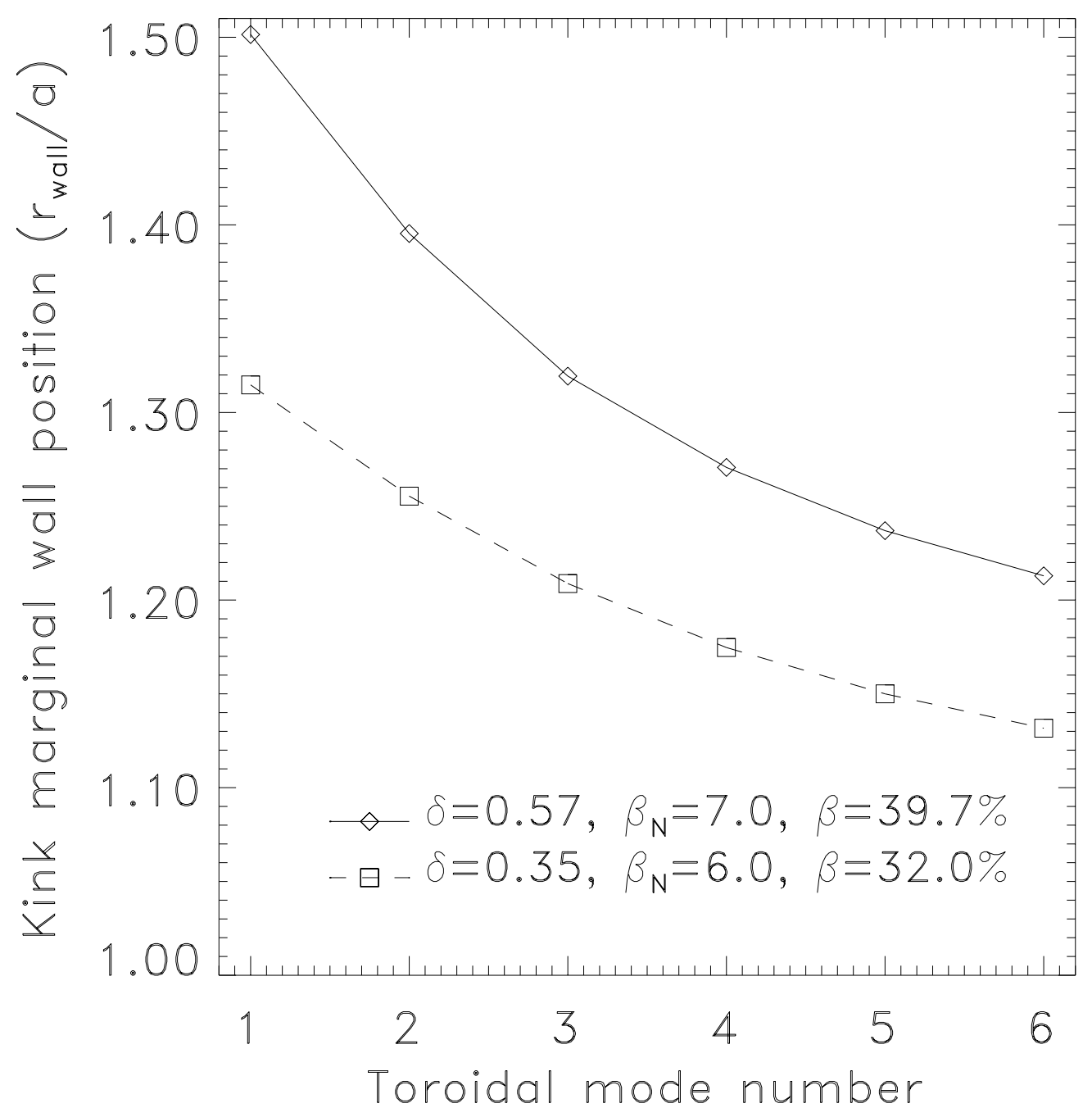

Figure 4: Conformal wall separation which marginally stabilizes $n=1-6$ kink modes as a function of toroidal mode number for ballooning stability optimized equilibria with $\mathrm{A}=1.6$, kappa=3.2, and triangularities 0.57 and 0.35 . 

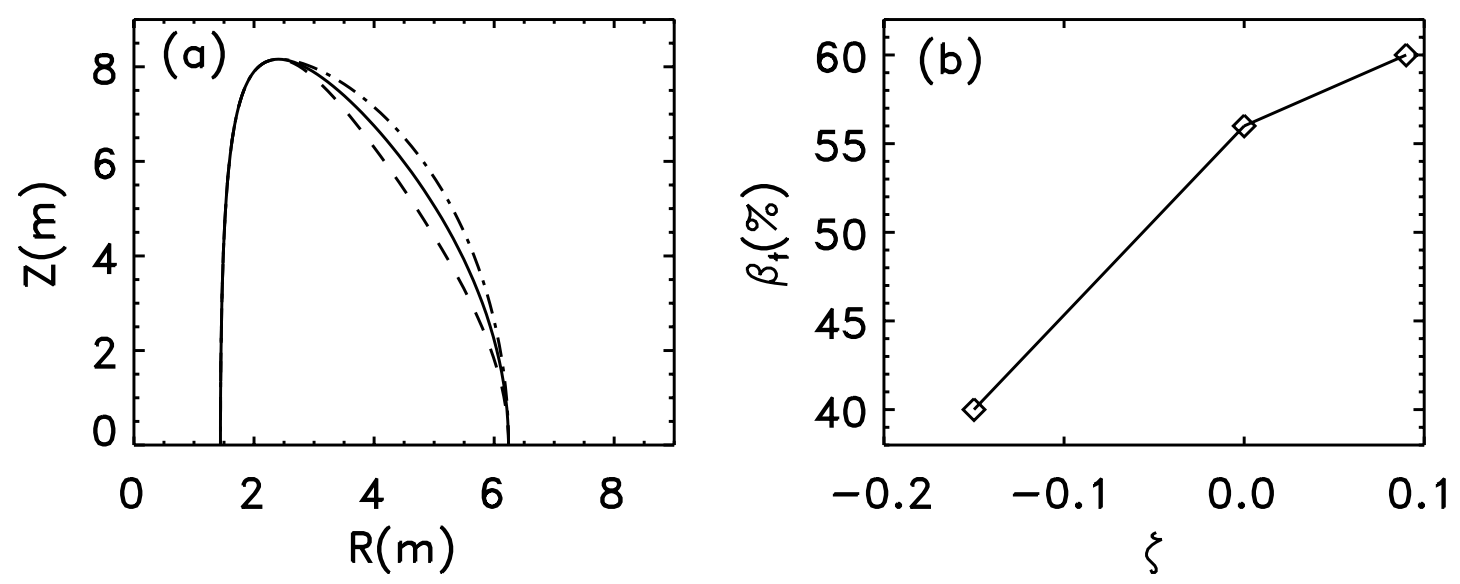

Figure 5: (a) Boundary shapes for the final ARIES-ST configuration ( $A=1.6, \kappa=3.4$, $\delta=0.64$ ) with outboard squareness values $\zeta_{\text {out }}=-0.1$ (dashed), 0.0 (solid), and +0.1 (dot-dash). (b) Impact of $\zeta_{\text {out }}$ on the ballooning marginal stability $\beta$ limit for the final ARIES-ST configuration. 

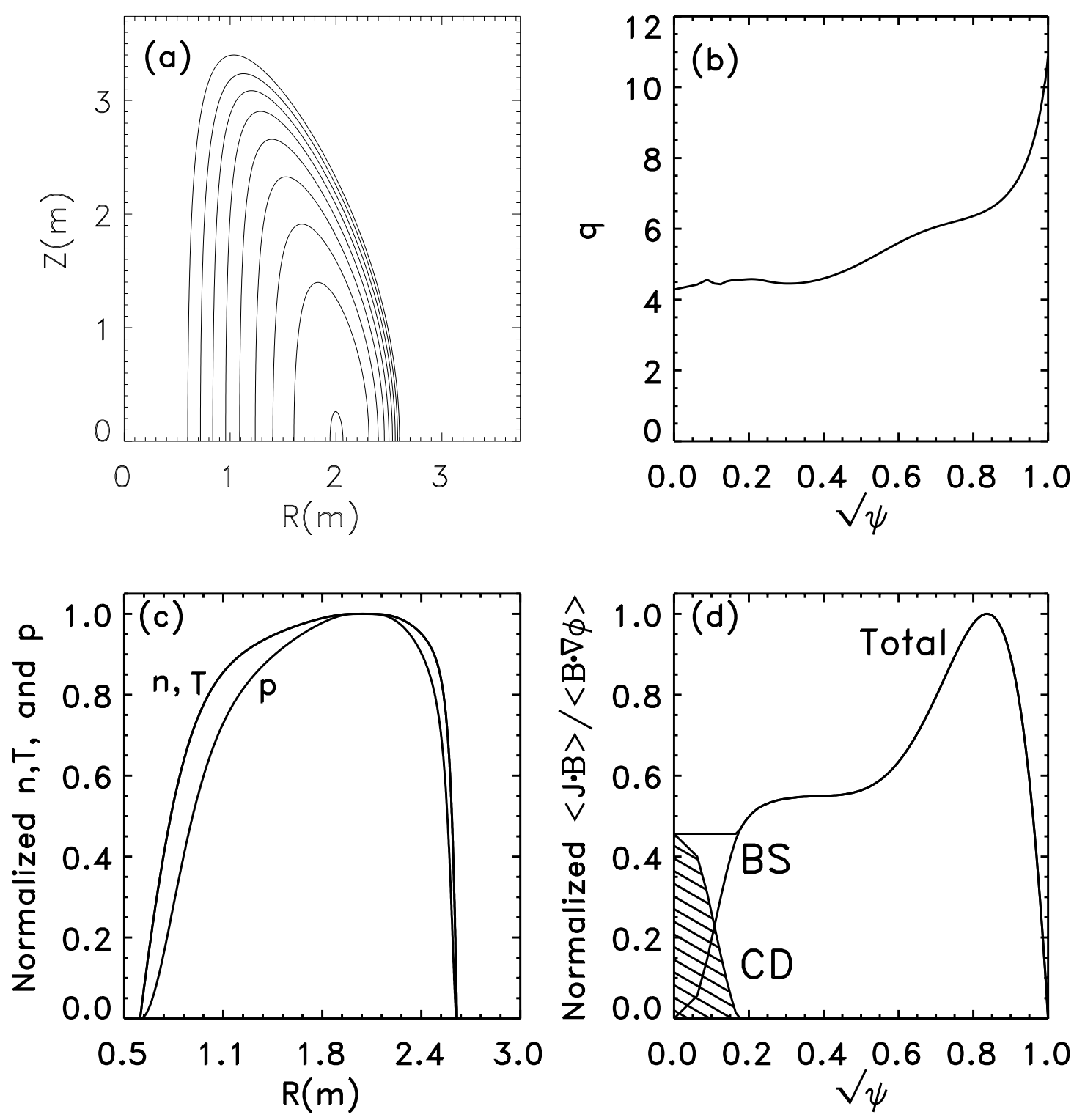

Figure 6: Equilibrium details of the final ARIES-ST configuration with $A=1.60$, $\kappa=3.4, \delta=0.64, \beta=56 \%$ and $\mathrm{f}_{\mathrm{BS}}>99 \%$ which is marginally stable to ballooning and $\mathrm{n}=1-6$ kink modes with a conducting wall at $\mathrm{r}_{\text {wall }} / \mathrm{a}=1.15$. ARIES-ST operating point has $\beta$ and beta $a_{N}$ reduced $10 \%$ below these values. (a) poloidal flux contours, (b) safety factor profile, (c) pressure, temperature, and density profiles, (d) total current, bootstrap current, and external current drive profiles 

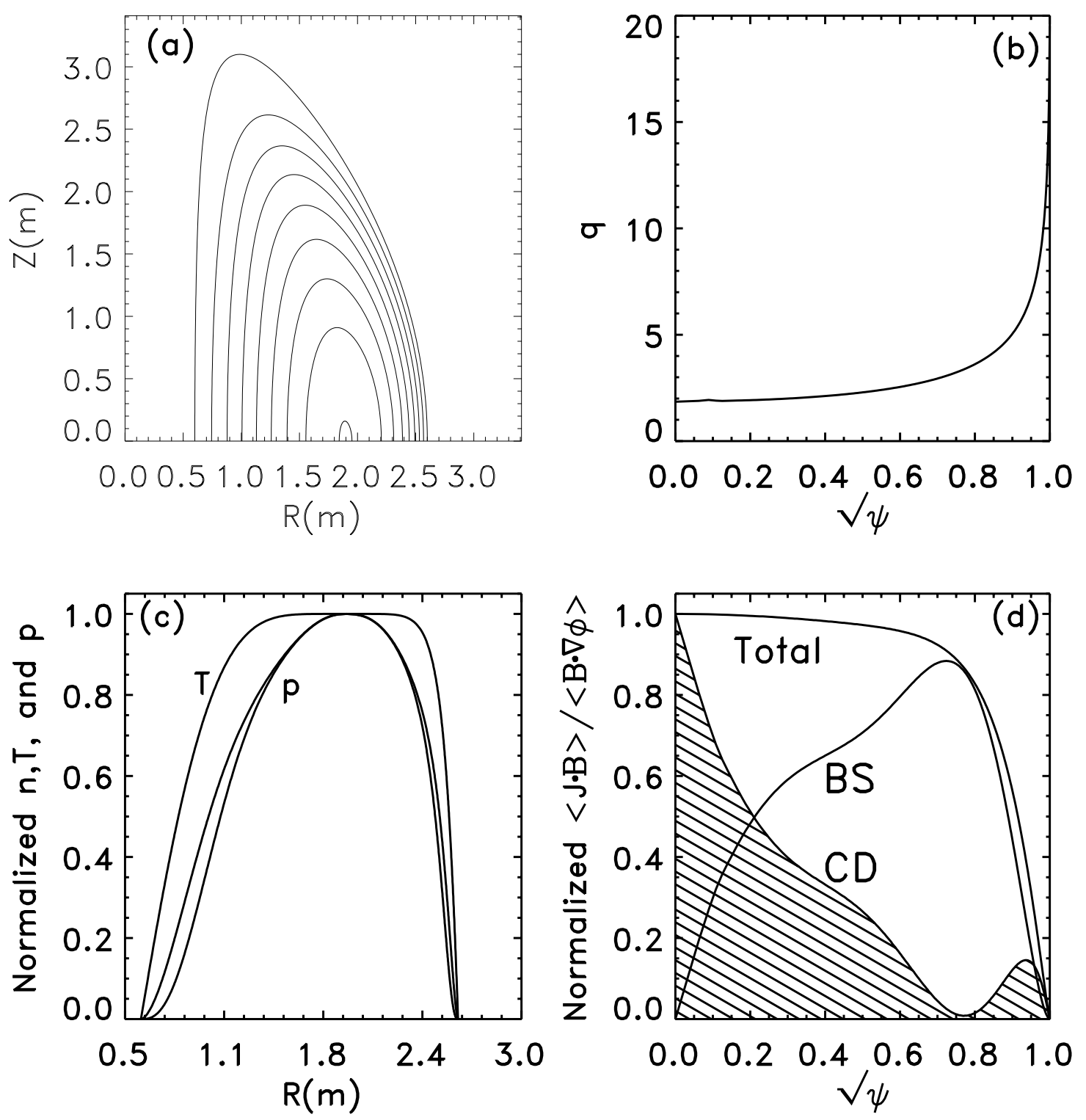

Figure 7: Details of an optimized ST equilibrium with $\mathrm{A}=1.60, \kappa=3.1, \delta=0.72, \beta$ $=29 \%$ and $\mathrm{f}_{\mathrm{BS}}=80 \%$ which is stable to ballooning and $\mathrm{n}=1-3$ kink modes without a conducting wall: (a) poloidal flux contours, (b) safety factor profile, (c) pressure, temperature, and density profiles, (d) total current, bootstrap current, and external current drive profiles 

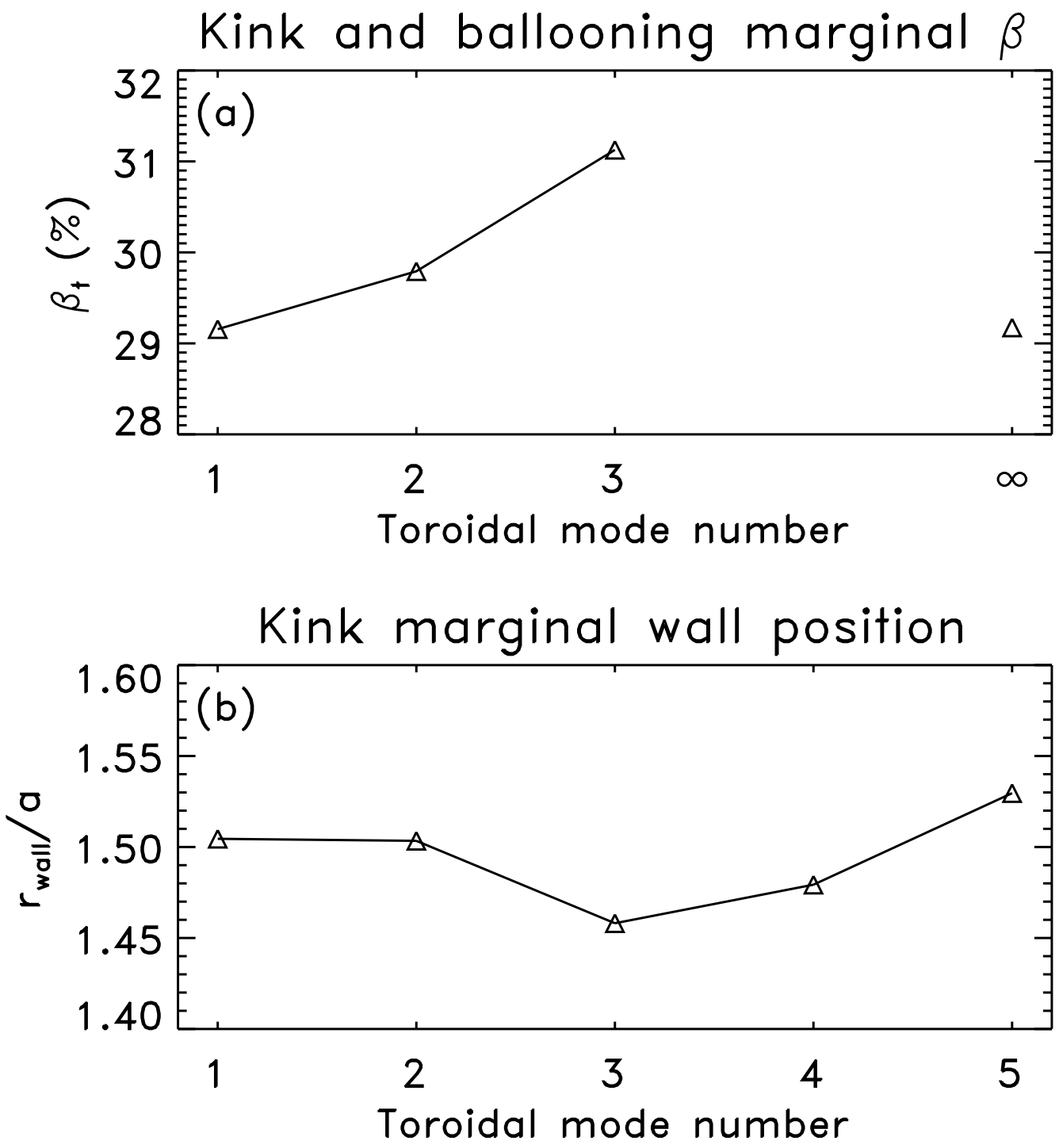

Figure 8: (a) No-wall kink and ballooning marginal $\beta$ for the equilibrium of Figure 7 (b) Kink marginal wall position for an equilibrium with the same geometric parameters, profiles, and $f_{\mathrm{BS}}$ as in Figure 6 , but with $\beta_{\mathrm{N}}\left(\beta_{t}\right)$ reduced to $5.5(25 \%)$. 


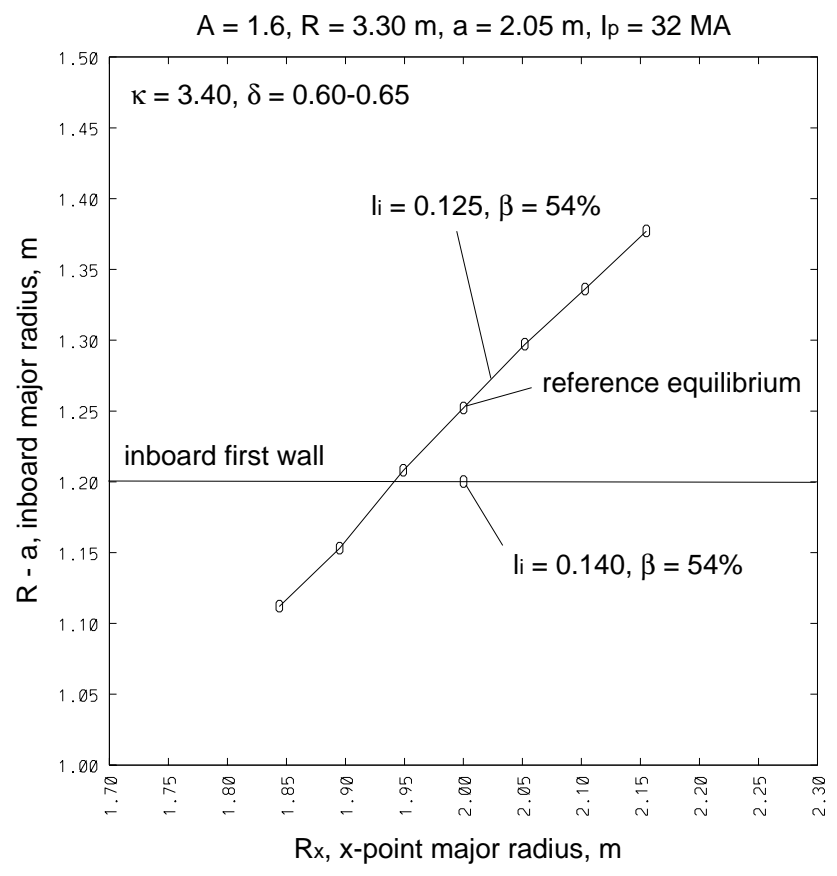

Figure 9: For a given internal inductive $l_{i}$ and beta, the inboard gap between the first wall and plasma boundary depends on the radial position of the x-point, $R_{x}$. Here, $\mathrm{A}=1.6, \mathrm{R}=3.30 \mathrm{~m}, \mathrm{a}=2.05 \mathrm{~m}, l_{p}=32 \mathrm{MA}$. 


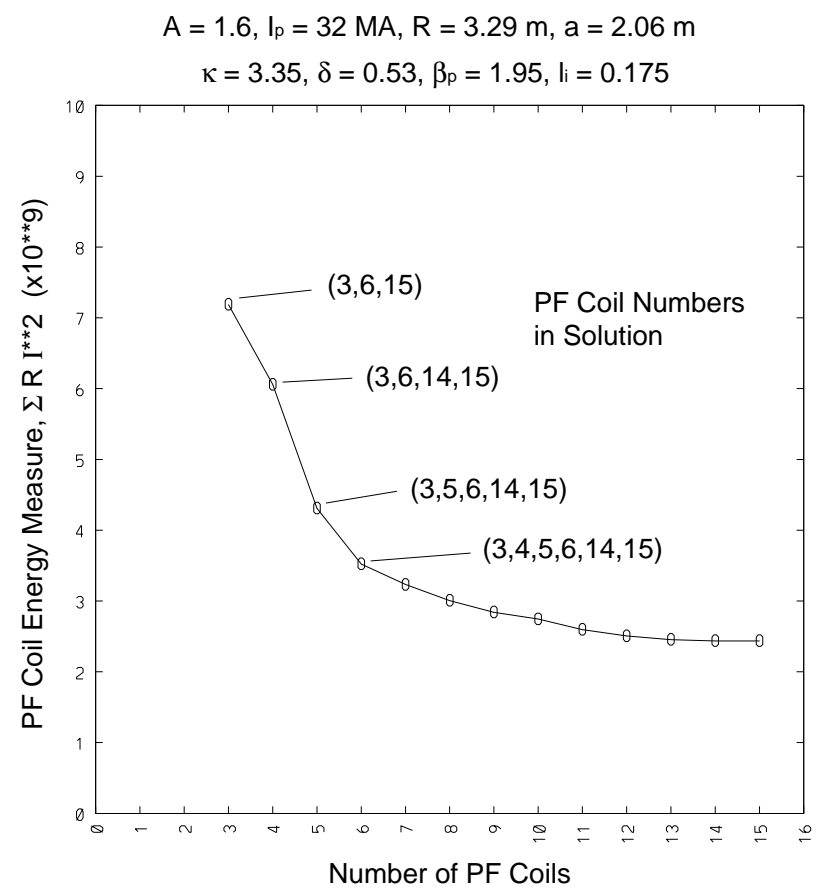

Figure 10: Dependence of the energy in the Poloidal Field (PF) System as a function of the number of PF coils used. Here, $A=1.6, l_{p}=32 \mathrm{MA}, R=3.29 \mathrm{~m}, a=2.05 \mathrm{~m}$, $\kappa=3.35, \delta=0.53, \beta-p=1.95, l_{i}=0.175$ 


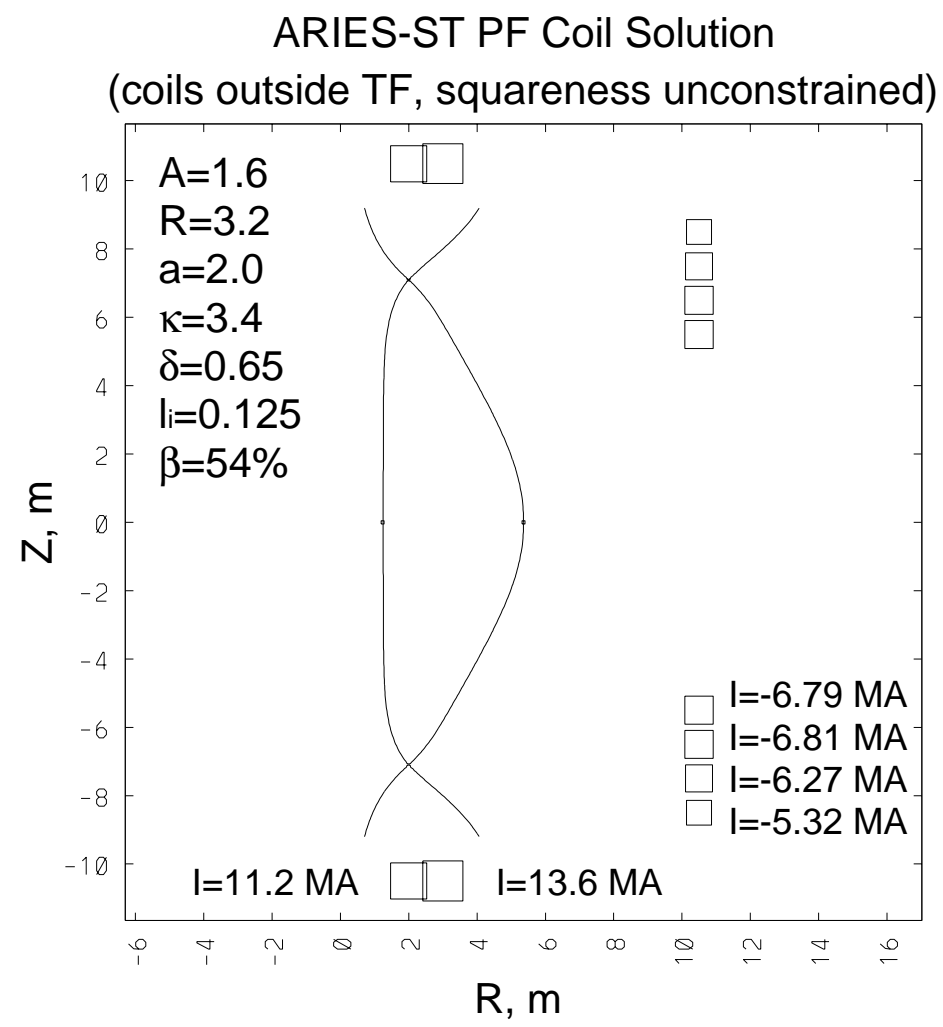

Figure 11: Plasma-vacuum interface for the reference 6-coil solution. 

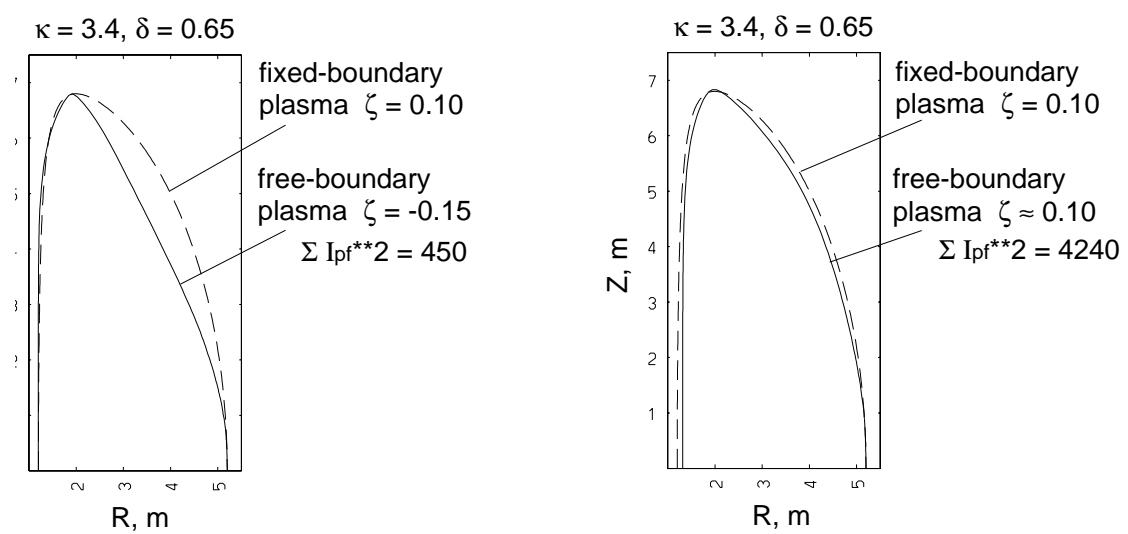

Figure 12: Comparison of free boundary solutions with values of the squareness parameter $\zeta$ of 0.15 and 0.10 . Note that the PF energy increases by nearly 10 for the larger squareness value. 


\section{Final ARIES-ST PF Coil Solution}

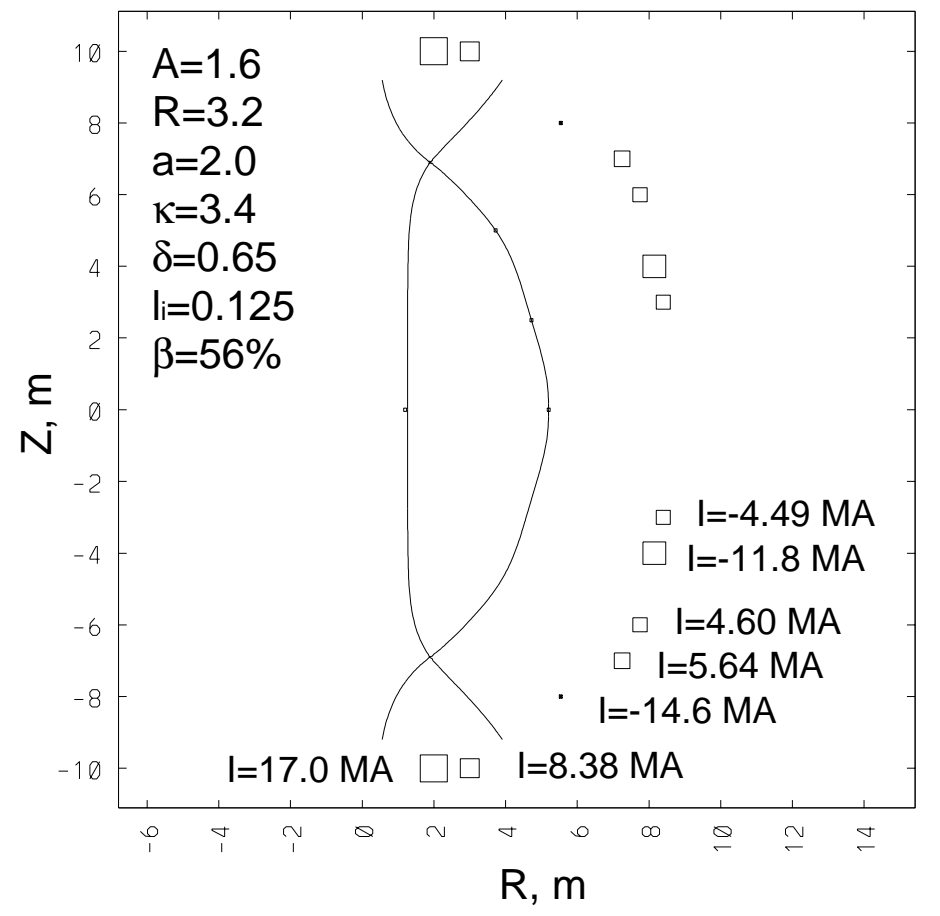

Figure 13: The final ARIES-ST free boundary solution sharing the plasma vacuum boundary and PF coil location and currents. 

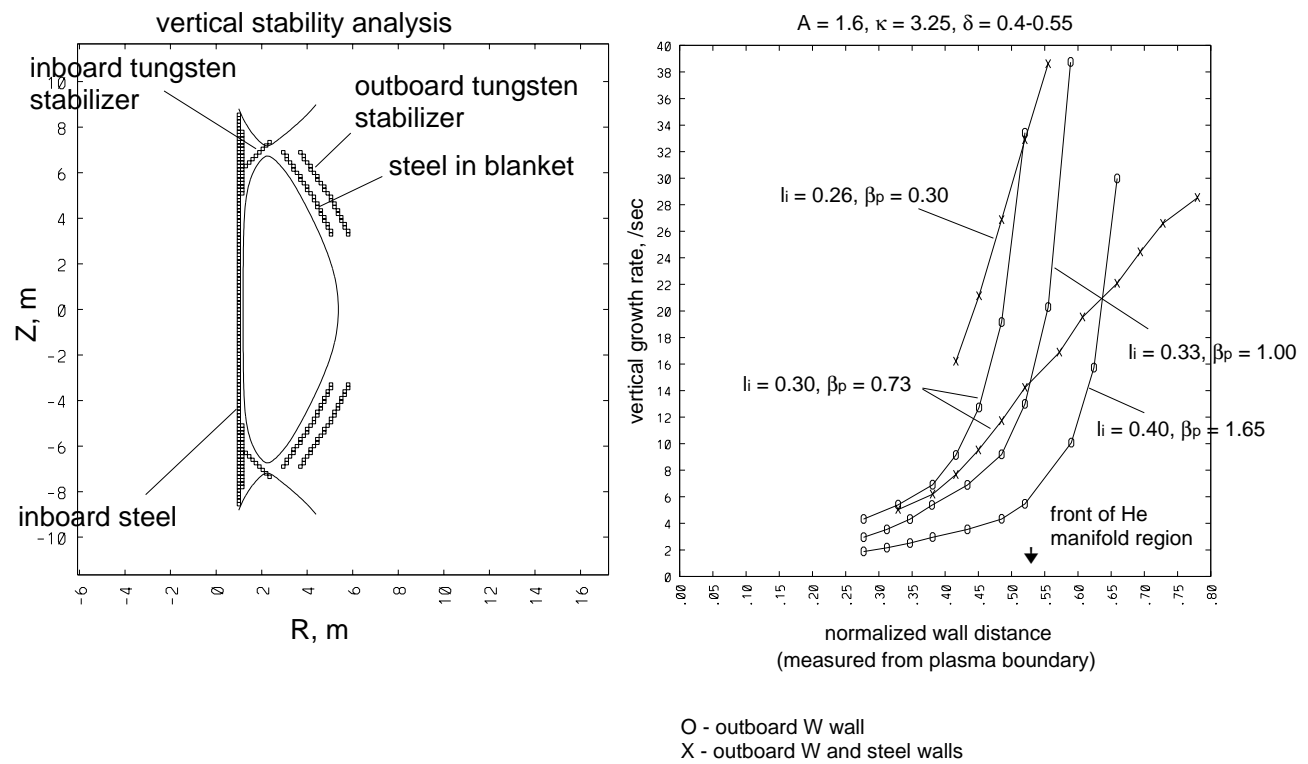

Figure 14: Structure model and growth rate results for several combinations of $\beta_{p}$ and $\ell_{i}$ 


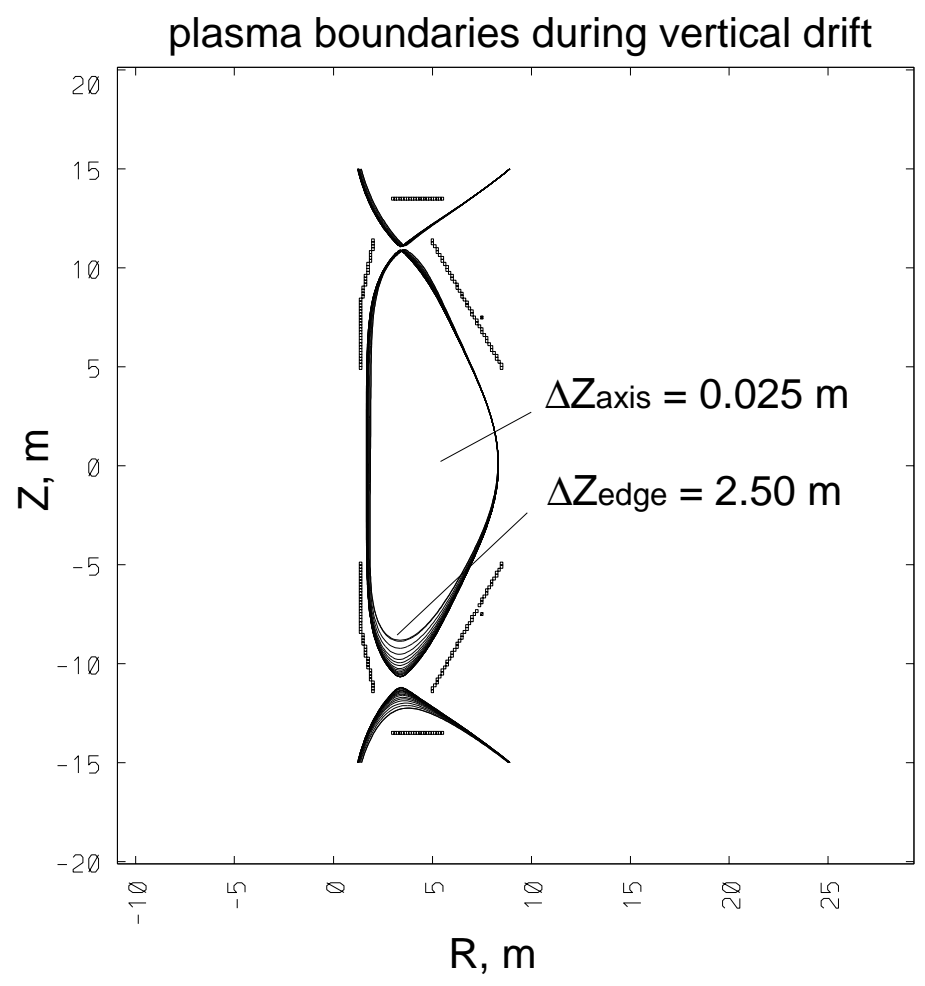

Figure 15: Sequence of plasma boundaries as the plasma is allowed to drift vertically with no feedback control. Notice the non-rigid behavior. 


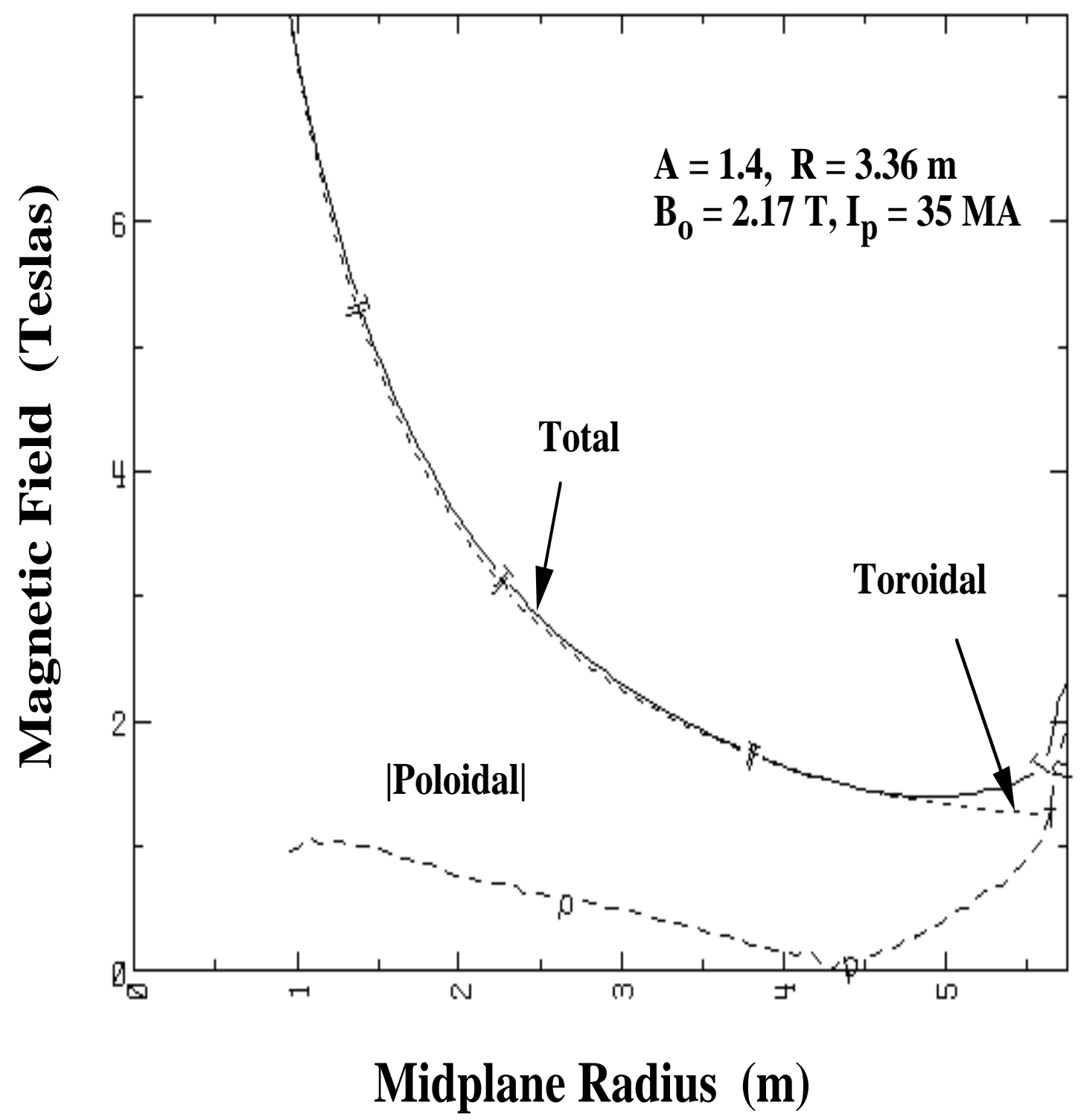

Figure 16: Magnitude of magnetic field along midplane major radius in a typical $\mathrm{ST}$ reactor plasma. 


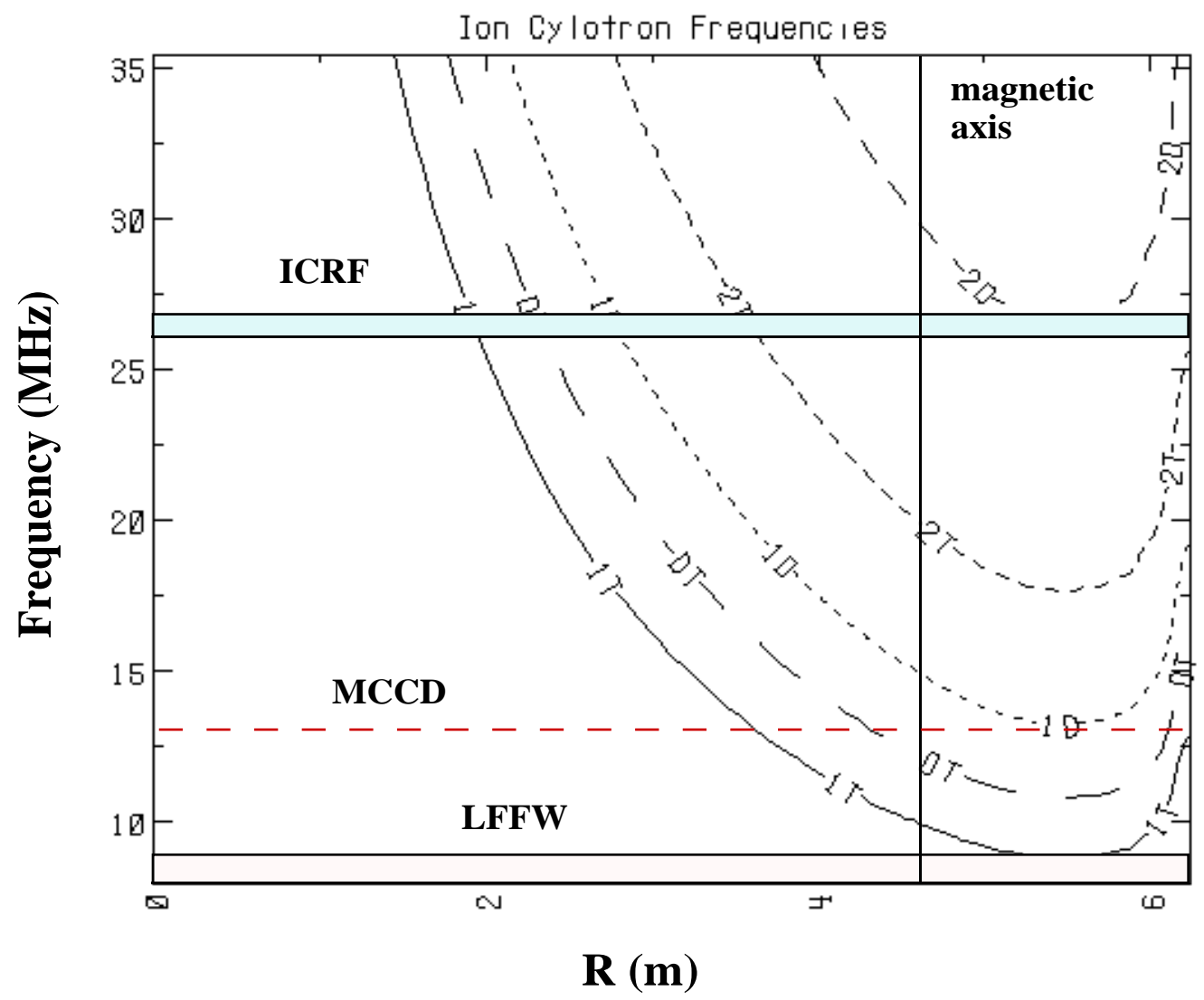

Figure 17: Selection of fast wave frequency for on-axis current drive in an ST reactor plasma. 

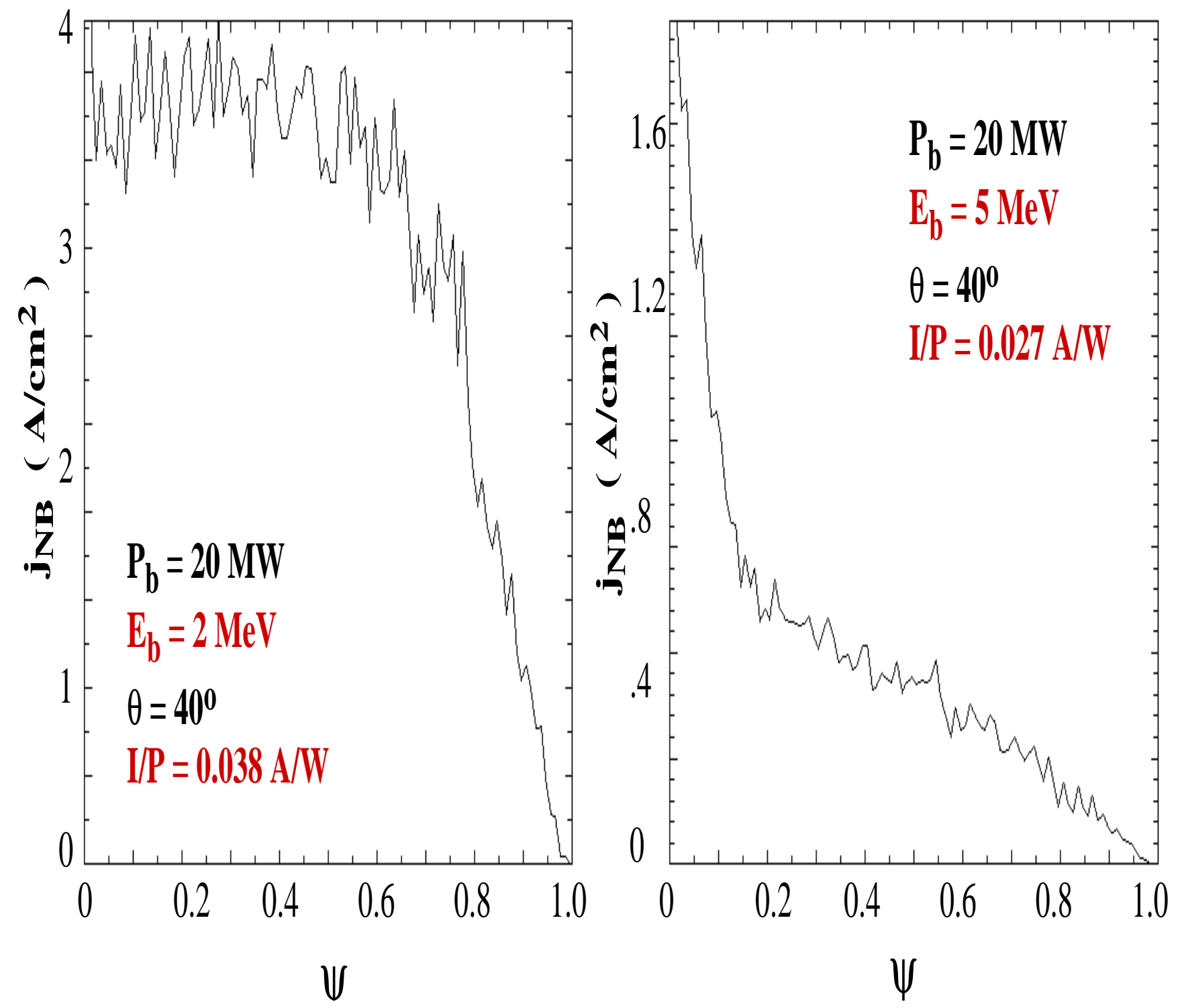

Figure 18: Neutral beam driven current profiles for a typical ST reactor plasma using a beam energy of (a) $2 \mathrm{MeV}$, and (b) $5 \mathrm{MeV}$. 


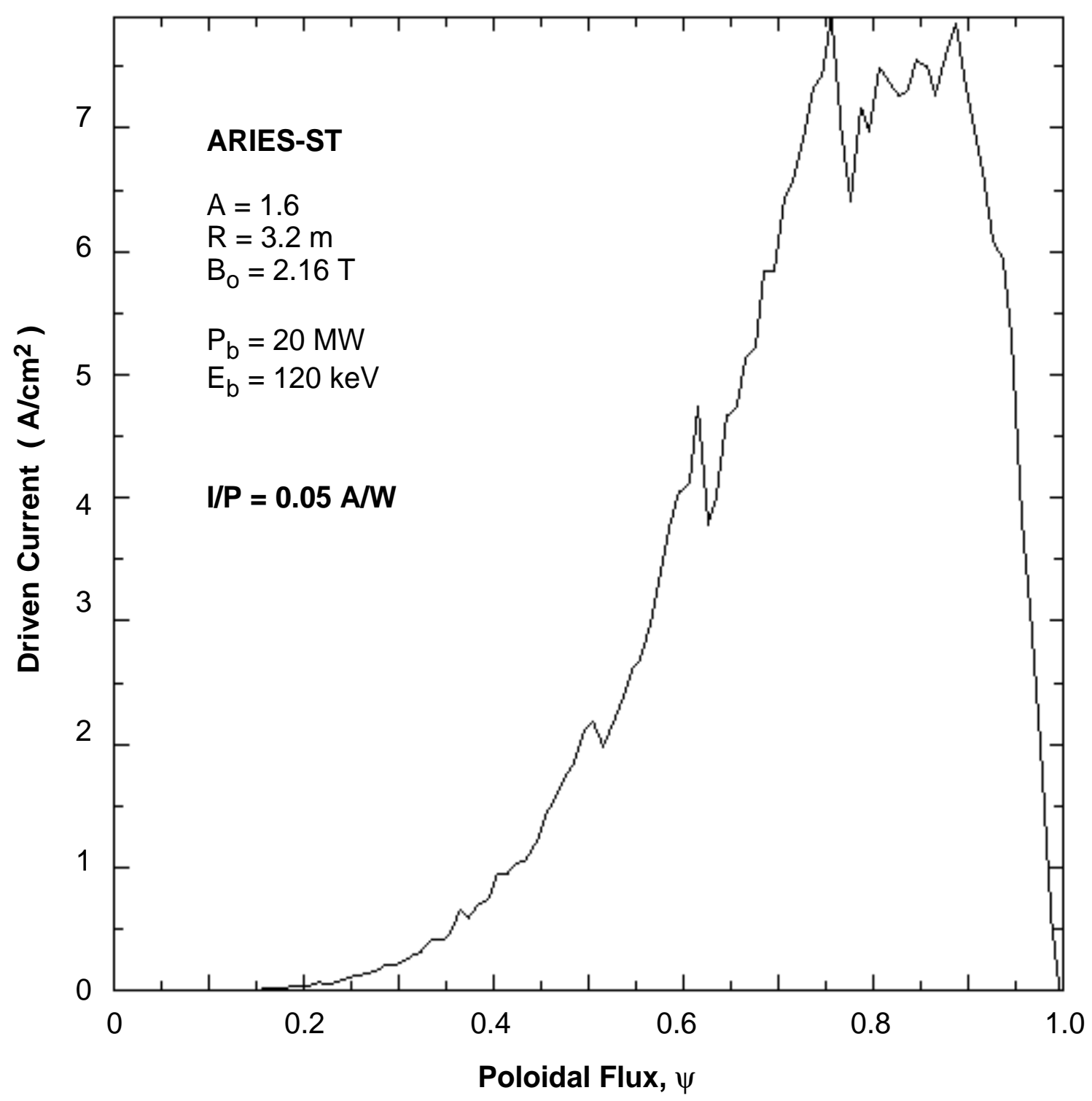

Figure 19: Off-axis neutral beam driven current profile for a typical ST reactor plasma using a beam energy of $120 \mathrm{keV}$. 


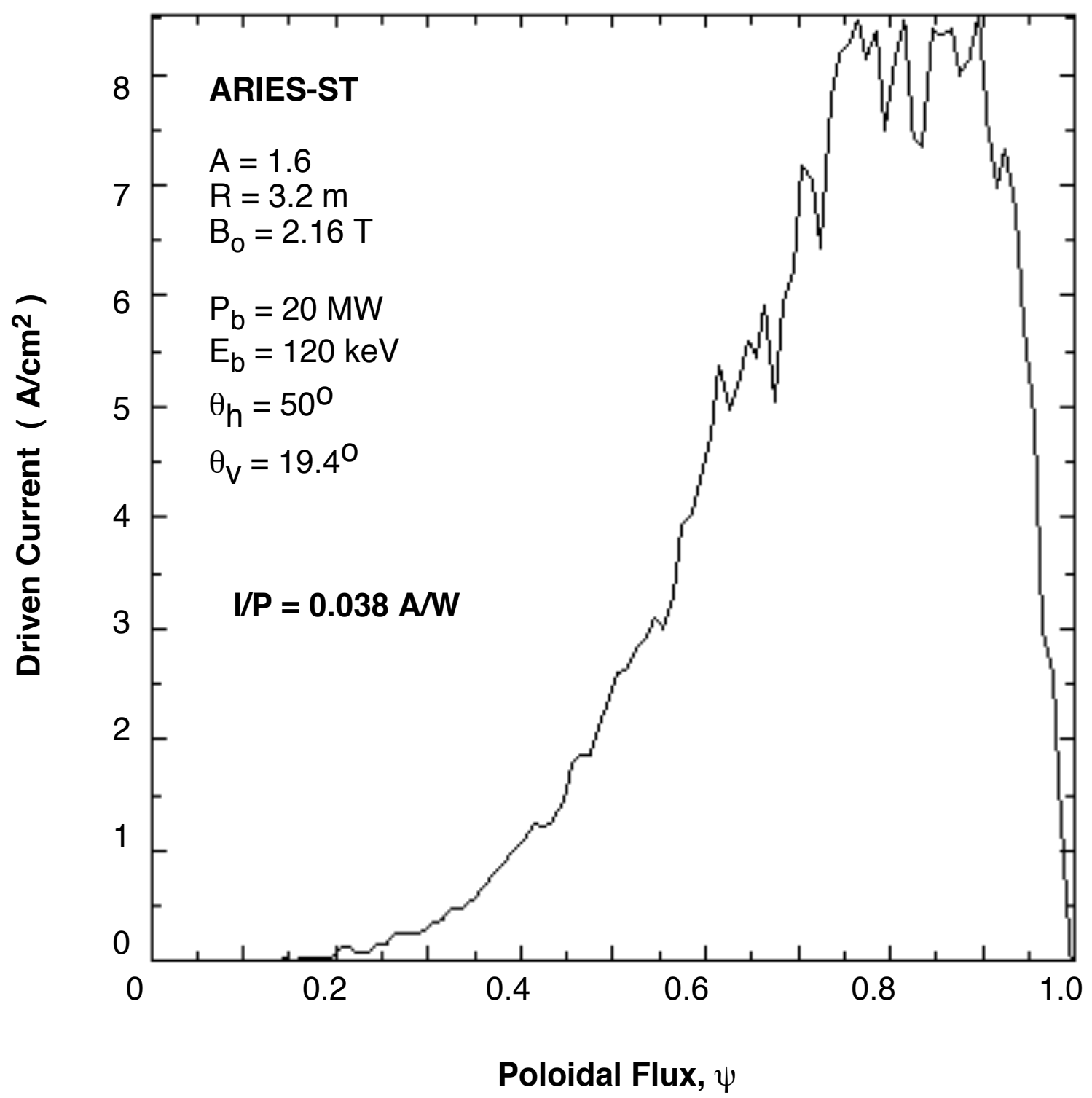

Figure 20: Off-axis neutral beam driven current profile in an ARIES-ST plasma using a beam energy of $120 \mathrm{keV}$ with three components. 


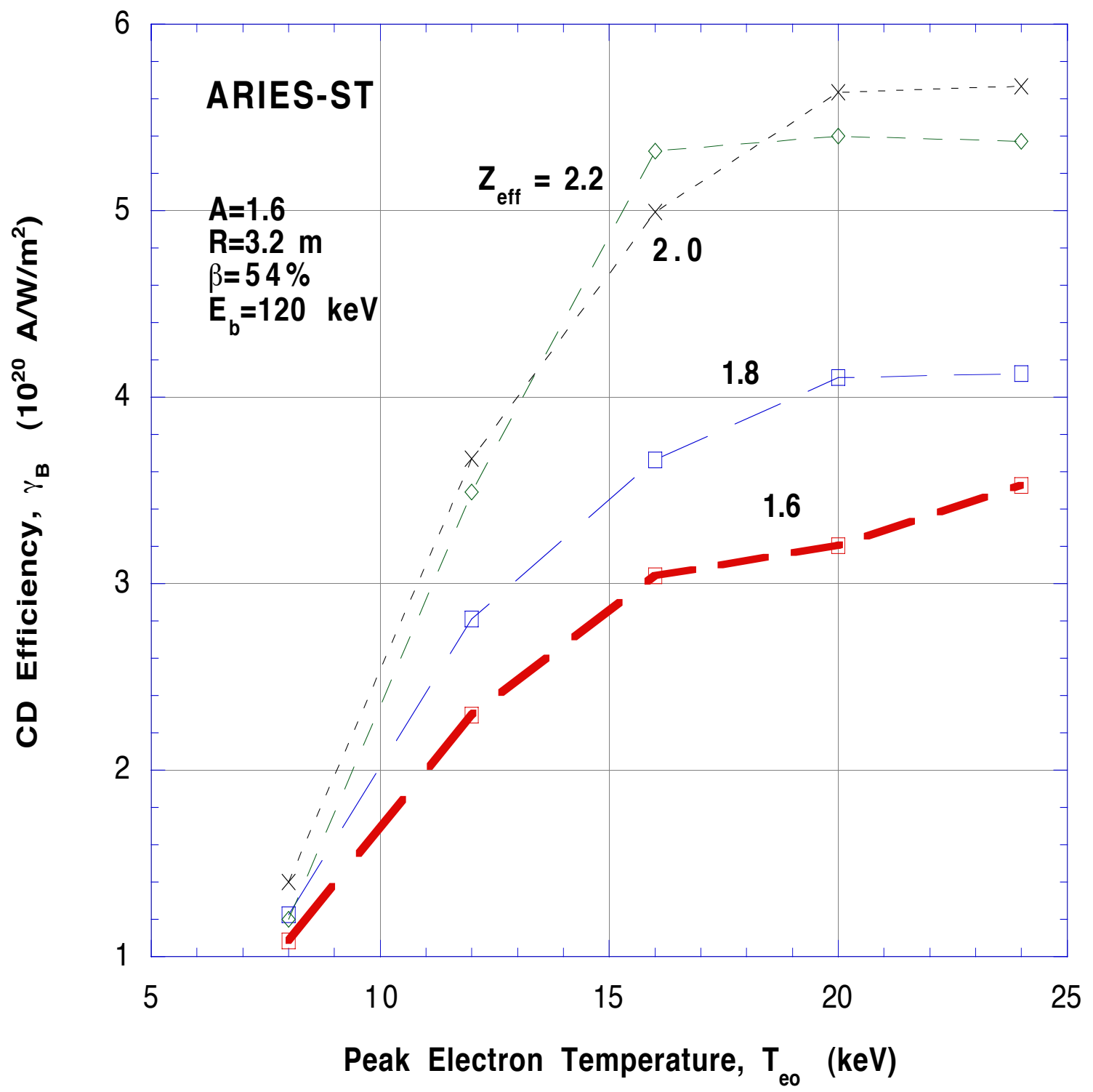

Figure 21: Calculated current drive efficiency as functions of electron temperature and plasma $Z_{\text {eff }}$, for ARIES-ST using $120 \mathrm{keV}$ neutral beams with current peaked at $\hat{\psi}=0.8$. 


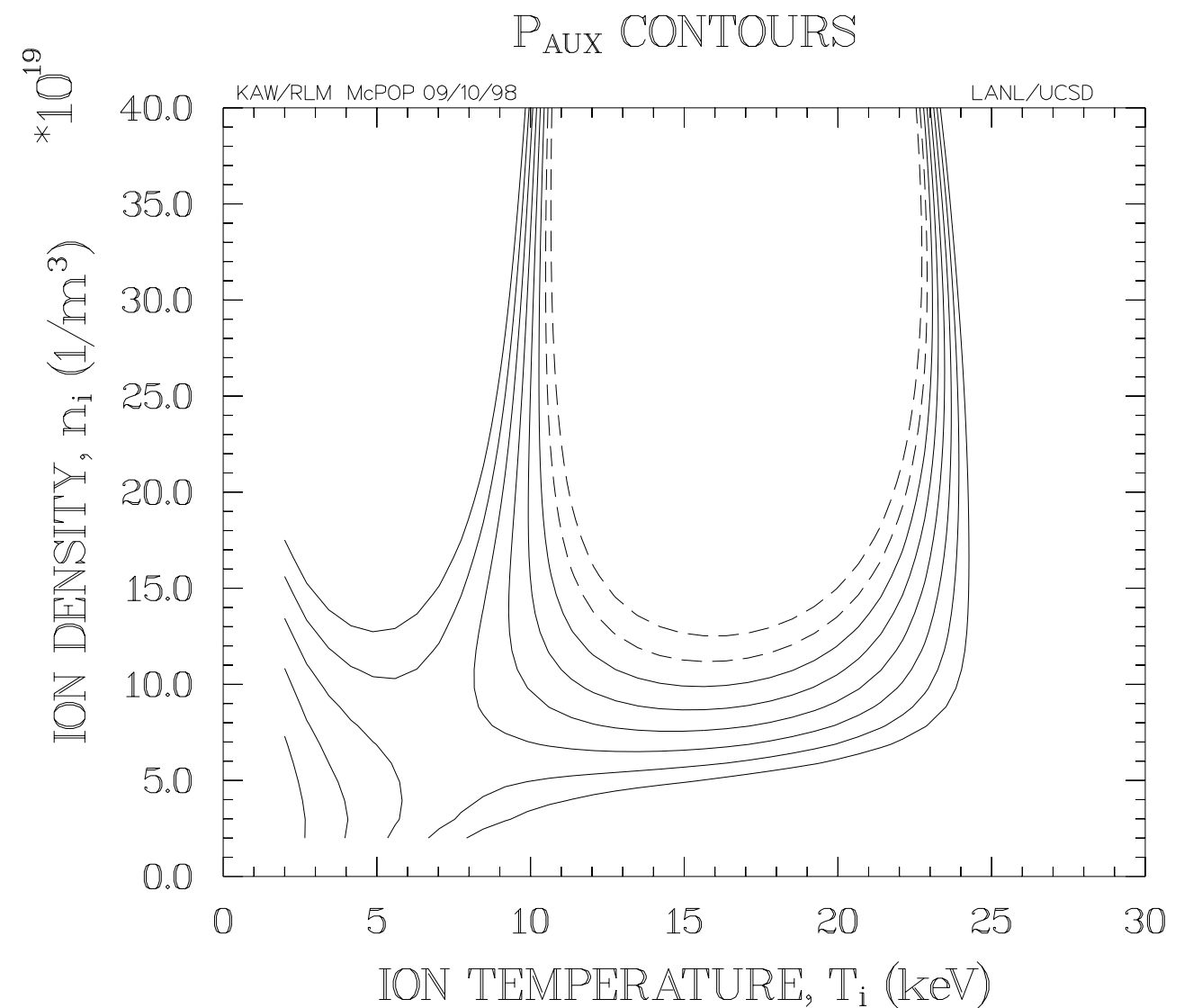

Figure 22: ARIES-ST Plasma Operating Contour (POPCON) diagram. Solid countours indicate auxiliary heating power, $P_{A U X}$, in increments of $10 \mathrm{MW}$ until "ignition" at the dashed contours. 

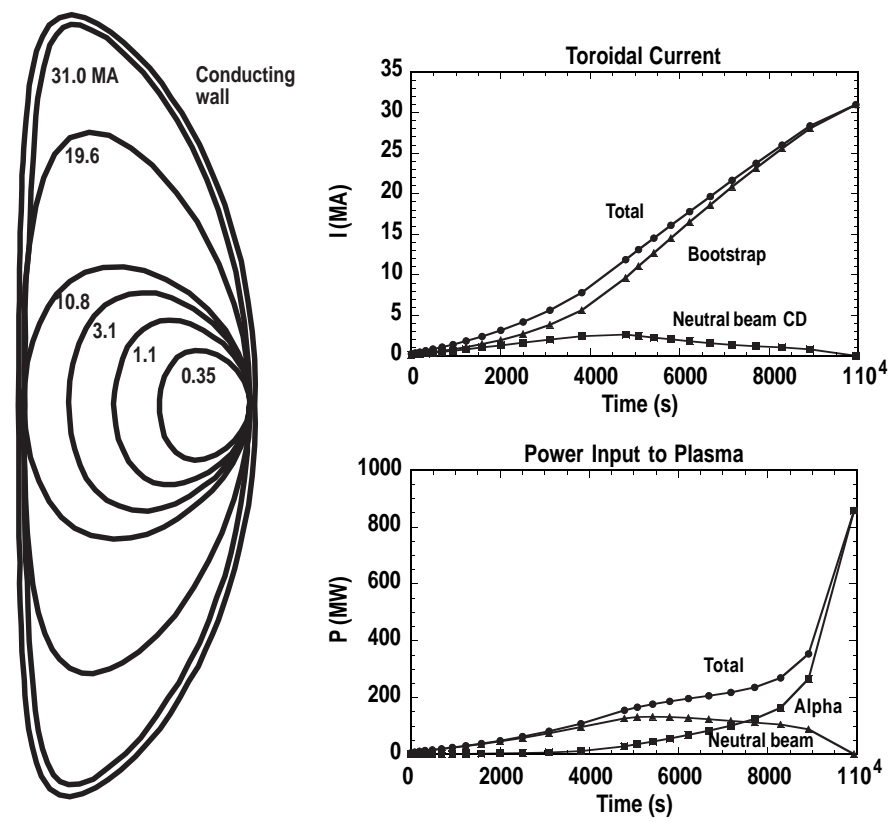

Figure 23: The minor radius and elongation are increased with the plasma current. Neutral beam current drive and power requirements are shown. 


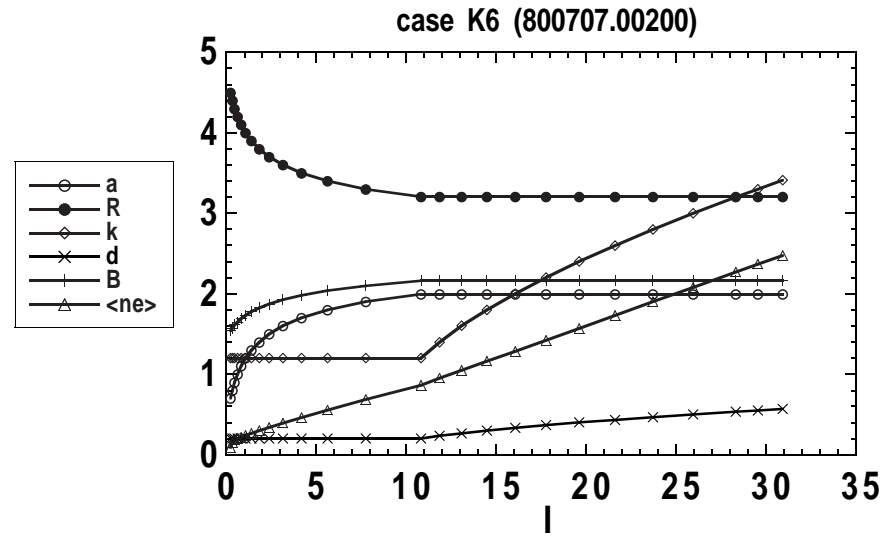

Figure 24: Geometrical quantities as a function of plasma current during bootstrap overdrive startup. 


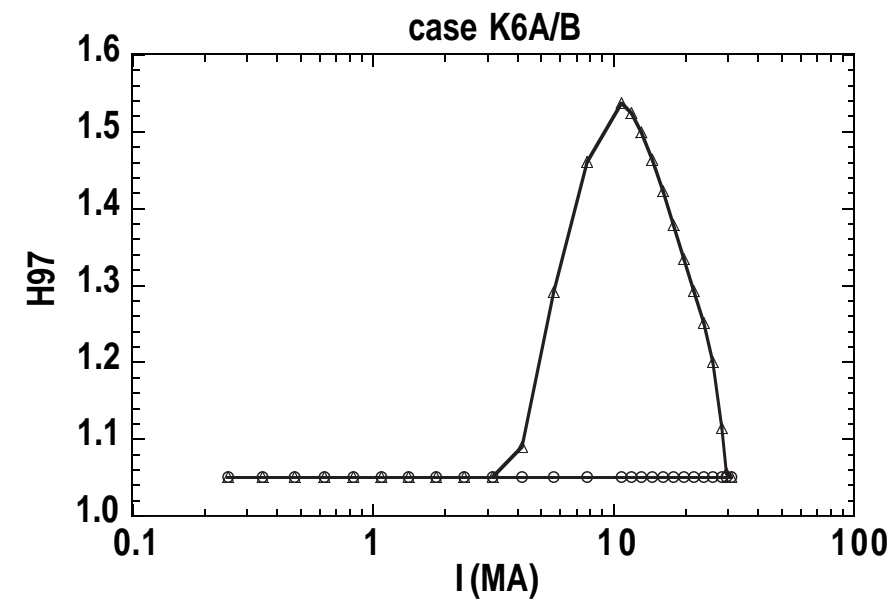

Figure 25: Required confinement multiplier for the two startup variants. 


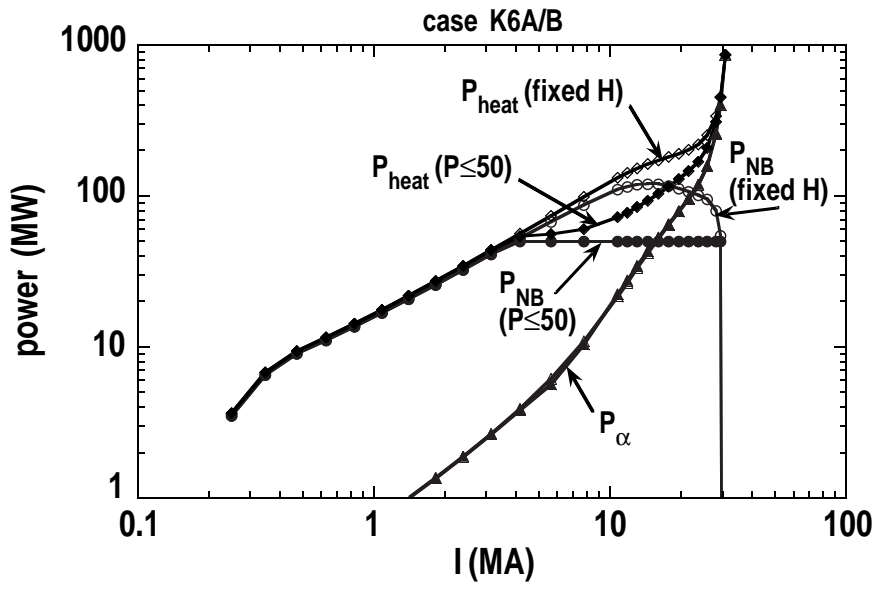

Figure 26: Log-Log plot of the power requirements for the two cases 


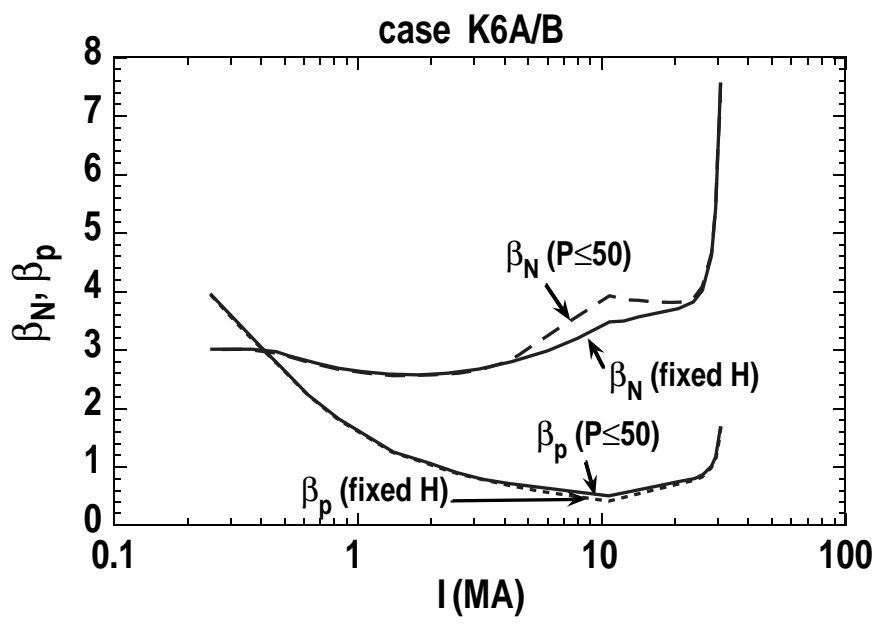

Figure 27: Stability parameters for the 2 startup variants. 


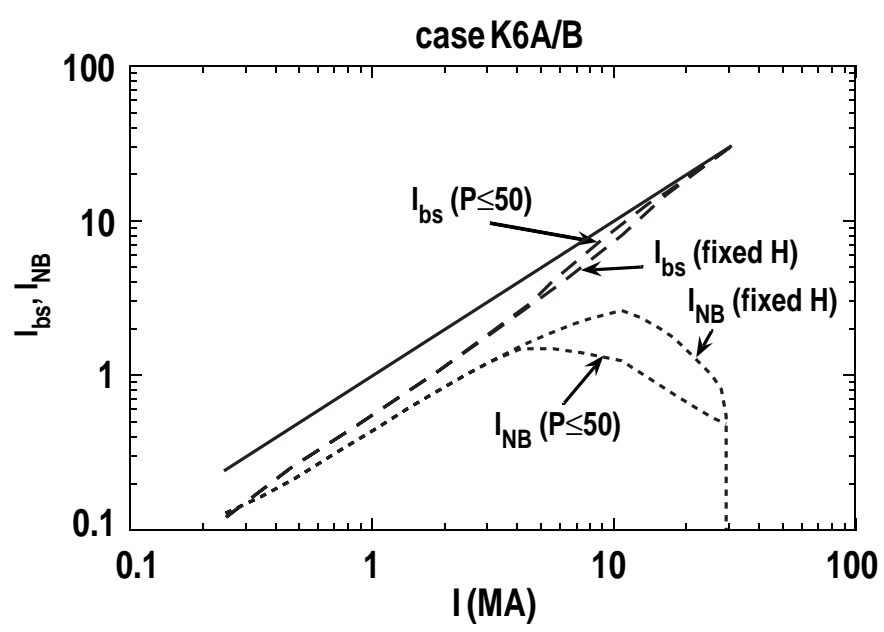

Figure 28: Log-Log plot of the current-drive requirements for the two cases. 